\title{
Rational Type Compatible Single-Valued Mappings via Unique Common Fixed Point Findings in Complex-Valued b-Metric Spaces with an Application
}

\author{
Shahid Mehmood, ${ }^{1}$ Saif Ur Rehman $\mathbb{D}^{1},{ }^{1}$ Naeem Jan $\mathbb{D}^{1},{ }^{1}$ Mabrook Al-Rakhami $\mathbb{D},{ }^{2}$ \\ and Abdu Gumaei ${ }^{3}{ }^{3}$ \\ ${ }^{1}$ Department of Mathematics, Gomal University, Dera Ismail Khan 29050, Pakistan \\ ${ }^{2}$ Research Chair of Pervasive and Mobile Computing, Department of Information Systems, King Saud University, \\ Riyadh 11543, Saudi Arabia \\ ${ }^{3}$ Computer Science Department, Faculty of Applied Sciences, Taiz University, Taiz 6803, Yemen
}

Correspondence should be addressed to Mabrook Al-Rakhami; malrakhami@ksu.edu.sa and Abdu Gumaei; abdugumaei@taiz.edu.ye

Received 3 April 2021; Accepted 7 May 2021; Published 26 May 2021

Academic Editor: Huseyin Isik

Copyright (c) 2021 Shahid Mehmood et al. This is an open access article distributed under the Creative Commons Attribution License, which permits unrestricted use, distribution, and reproduction in any medium, provided the original work is properly cited.

\begin{abstract}
In this paper, we establish some new generalized rational type common fixed point results for compatible three self-mappings in complex-valued b-metric space, in which a one self-map is continuous. In support of our results, we present some illustrative examples to verify the validity of our main work. Moreover, we present the application of two Urysohn integral type equations (UITEs) for the existence of a common solution to support our work. The UITEs are $v_{1}(p)=\int_{k_{1}}^{k_{2}} Q_{1}\left(p, r, v_{1}(r)\right) d r+\hbar_{1}(p)$ and $v_{2}($ $p)=\int_{k_{1}}^{k_{2}} Q_{2}\left(p, r, v_{2}(r)\right) d r+\hbar_{2}(p)$, where $p \in\left[k_{1}, k_{2}\right], v_{1}, v_{2}, \hbar_{1}, \hbar_{2} \in V$, where $V=C\left(\left[k_{1}, k_{2}\right], \mathbb{R}^{n}\right)$ is the set of all real-valued continuous functions defined on $\left[k_{1}, k_{2}\right]$ and $Q_{1}, Q_{2}:\left[k_{1}, k_{2}\right] \times\left[k_{1}, k_{2}\right] \times \mathbb{R}^{n} \longrightarrow \mathbb{R}^{n}$.
\end{abstract}

\section{Introduction}

The theory of fixed point is one of the most interesting area of research in Mathematics. Initially, the concept of this theory was given by Banach [1] and he proved "a Banach contraction theorem for fixed point" which is stated as "a singlevalued contractive type mapping in a complete metric space has a unique fixed point." After the publication of this research article, many authors have contributed their ideas to the theory of fixed point in the context of metric spaces and proved different contractive type fixed point results for single-valued and multivalued mappings with different types of applications. Some fixed soft points and a-fixed soft points results can be found in $[2,3]$.

The concept of b-metric space was first introduced by Bakhtin [4], while Czerwik [5] proved some fixed point results for nonlinear set-valued contractive type mappings in b-metric spaces. Later on, Akkouchi [6] established some common fixed point theorems (CFP-theorems) for singlevalued mappings under an implicit relation in b-metric spaces. In [7], Aghajani et al. proved CFP-results under the generalized weak contraction in partially ordered b-metric spaces. Further, Aydi et al. [8, 9] presented some FPtheorems and CFP-theorems for set-valued quasicontraction and weak $\phi$-contraction, respectively, in bmetric spaces. In 2013, Roshan et al. [10] established some generalized contractive type CFP-theorems in b-metric spaces and they proved that the b-metric function used in the theorems and results are not necessarily continuous. Some more FP-results in b-metric space can be found in [11-19]; the references are therein.

In 2011, Azam et al. [20] introduced the notion of complex-valued metric space and proved some CFPtheorems for a pair of self-mappings. Though complex- 
valued metric space forms a special class of cone metric space, so far this concept is proposed to define rational type expressions that are not significant in cone metric spaces, and therefore, some results of the analysis cannot be generalized to cone metric spaces. Properly the notion of complexvalued metric space was introduced by Rouzkard and Imdad [21] which generalized the expression of Azam et al. [20] and proved some CFP-theorems. Some more FP-results in the context of complex-valued metric spaces can be found in [22-24].

In 2013, Rao et al. [25] introduced the notion of complexvalued b-metric space which generalized the notion of complex-valued metric spaces given by Azam et al. [20] in 2011. They presented some CFP-results for generalized contraction conditions in complex-valued b-metric space. Later on, Mukheimer [26] extended and improved the results of $[20,25]$ and established some unique CFP-theorems in complex-valued metric spaces with illustrative examples.

In this paper, we establish some new generalized rational type CFP-theorems for compatible three self-mappings on complex-valued b-metric spaces in which one is a continuous self-map. Our results extend and modify many results given in the literature. This paper is organized as follows: Section 2 consists of preliminary concepts. In Section 3, we present some generalized unique CFP-theorems for compatible three self-mappings in complex-valued b-metric spaces with some illustrative examples to verify the validity of our work. In Section 4, we present an application of the two UITEs for the existence of a common solution to support our main result, while in Section 5, we discuss the conclusion.

\section{Preliminaries}

Consider $\mathbb{C}$ represents a set of complex numbers and $z_{i}, z_{i i}$ $\in \mathbb{C}$. Define $\leq$ as $z_{i} \leq z_{i i}$, iff $R_{e}\left(z_{i}\right) \leq R_{e}\left(z_{i i}\right)$ and $I_{m}\left(z_{i}\right) \leq I_{m}($ $\left.z_{i i}\right)$, where $R_{e}$ denotes the real part and $I_{m}$ denotes the imaginary part of a complex number. Accordingly, $z_{i} \leq z_{i i}$, if any one of the following conditions holds:

$\left(C_{1}\right) R_{e}\left(z_{i}\right)=R_{e}\left(z_{i i}\right)$ and $I_{m}\left(z_{i}\right)=I_{m}\left(z_{i i}\right)$,

$\left(C_{2}\right) R_{e}\left(z_{i}\right)<R_{e}\left(z_{i i}\right)$ and $I_{m}\left(z_{i}\right)=I_{m}\left(z_{i i}\right)$,

$\left(C_{3}\right) R_{e}\left(z_{i}\right)=R_{e}\left(z_{i i}\right)$ and $I_{m}\left(z_{i}\right)<I_{m}\left(z_{i i}\right)$,

$\left(C_{4}\right) R_{e}\left(z_{i}\right)<R_{e}\left(z_{i i}\right)$ and $I_{m}\left(z_{i}\right)<I_{m}\left(z_{i i}\right)$,

In particular, we can write $z_{i} \leqslant z_{i i}$ if $z_{i} \neq z_{i i}$ and one of $\left(C_{2}\right)$, $\left(C_{3}\right)$, and $\left(C_{4}\right)$ is satisfied.

Remark 1 (see [26]). The properties given below hold and can be verified:

$\left(R_{1}\right)$ if $a_{1}, a_{2} \in \mathbb{R}$ and $a_{1} \leq a_{2} \Rightarrow a_{1} y \leq a_{2} y \forall y \in \mathbb{C}$,

$\left(R_{2}\right) 0 \leq z_{i} \leq z_{i i} \Rightarrow\left|z_{i}\right|<\left|z_{i i}\right|$,

$\left(R_{3}\right) z_{i} \leq z_{i i}$ and $z_{i i}<z_{i i i} \Rightarrow z_{i}<z_{i i i}$.

Definition 2 (see [5]). Let $V$ be a nonempty set and let $b \geq 1$ be a given real number. A function $\delta: V \times V \longrightarrow[0, \infty)$ is said to be a b-metric on $V$ if it holds the following conditions:

$\left(b_{m} 1\right) \delta\left(v_{1}, v_{2}\right)=0 \Leftrightarrow v_{1}=v_{2}$,

$\left(b_{m}\right) \delta\left(v_{1}, v_{2}\right)=\delta\left(v_{2}, v_{1}\right)$,

$\left(b_{m} 3\right) \delta\left(v_{1}, v_{2}\right) \leq b\left[\delta\left(v_{1}, v_{3}\right)+\delta\left(v_{3}, v_{2}\right)\right]$,

for all $v_{1}, v_{2}, v_{3} \in V$. The pair $(V, \delta)$ is called a b-metric space, where $b$ is a coefficient of $(V, \delta)$.
Definition 3 (see [25]). Let $V$ be a nonempty set and let $b \geq 1$ be a given real number. A function $\delta: V \times V \longrightarrow \mathbb{C}$ is said to be a complex-valued b-metric on $V$ if it holds the following conditions:

$\left(C b_{m} 1\right) \delta\left(v_{1}, v_{2}\right) \geq 0$ and $\delta\left(v_{1}, v_{2}\right)=0$ if and only if $v_{1}=$ $v_{2}$,

$\left(C b_{m} 2\right) \delta\left(v_{1}, v_{2}\right)=\delta\left(v_{2}, v_{1}\right)$

$\left(C b_{m} 3\right) \delta\left(v_{1}, v_{2}\right) \leq b\left[\delta\left(v_{1}, v_{3}\right)+\delta\left(v_{3}, v_{2}\right)\right]$

for all $v_{1}, v_{2}, v_{3} \in V$. The pair $(V, \delta)$ is called a complexvalued b-metric space, where $b$ is a coefficient of $(V, \delta)$.

Example 1. Let $V=[0, \infty)$. The mapping $\delta: V \times V \longrightarrow \mathbb{C}$ is defined by

$$
\delta\left(v_{1}, v_{2}\right)=\frac{7}{17}\left|v_{1}-v_{2}\right|^{2}+i \frac{7}{17}\left|v_{1}-v_{2}\right|^{2}, \forall v_{1}, v_{2} \in \mathbb{V} .
$$

Then $(V, \delta)$ is a complex-valued b-metric space with $b=2$.

Definition 4 (see $[25,26])$. Let $(V, \delta)$ is a complex-valued bmetric space and $\left\{v_{n}\right\}$ be a sequence in $V$ and $v \in V$. Consider the following:

(1) If there is $N_{1} \in \mathbb{N}$ for every $c_{1} \in \mathbb{C}$ and $0<c_{1}$ such that for all $n>N_{1}, \delta\left(v_{n}, v\right)<c_{1}$, then $\left\{v_{n}\right\}$ is called convergent, $\left\{v_{n}\right\}$ converges to $v$, and $v$ is a limit point of $\left\{v_{n}\right\}$. Mathematically, it can be written as $\lim _{n \longrightarrow \infty}$ $v_{n}=v$ or $\left\{v_{n}\right\} \longrightarrow v$ as $n \longrightarrow \infty$.

(2) If there is $N_{1} \in \mathbb{N}$ for every $c_{1} \in \mathbb{C}$ and $0<c_{1}$ such that for all $n>N_{1}, \delta\left(v_{n}, v_{n+m}\right)<c_{1}$, where $m \in \mathbb{N}$, then $\left\{v_{n}\right\}$ is said to be Cauchy sequence.

(3) If every Cauchy sequence is convergent, then $(V, \delta)$ is said to be complete complex-valued b-metric space.

Lemma 5 (see $[25,26])$. Let $(V, \delta)$ be a complex-valued $b$ metric space and let $\left\{v_{n}\right\}$ be a sequence in $V$. Then, $\left\{v_{n}\right\}$ converges to viff $\left|\delta\left(v_{n}, v\right)\right| \longrightarrow 0$ as $n \longrightarrow \infty$.

Lemma 6 (see $[25,26])$. Let $(V, \delta)$ be a complex-valued $b$ metric space and let $\left\{v_{n}\right\}$ be a sequence in $V$. Then, $\left\{v_{n}\right\}$ is a cauchy sequence if $f\left|\delta\left(v_{n}, v_{n+m}\right)\right| \longrightarrow 0$ as $n \longrightarrow \infty$.

To prove the main result, we will use following lemma.

Lemma 7 (see [10]). Let $(V, \delta)$ be a complex-valued b-metric space. Consider $\left\{v_{n}\right\}$ and $\left\{w_{n}\right\}$ be two sequences such that $\lim _{n \longrightarrow \infty}\left|\delta\left(v_{n}, w_{n}\right)\right|=0$, whenever $\left\{v_{n}\right\}$ is a sequence in $V$ such that $\lim _{n \longrightarrow \infty} v_{n}=z$ for some $z \in V$; then, $\lim _{n \longrightarrow \infty} w_{n}=z$.

Proof. Given that

$$
\lim _{n \longrightarrow \infty}\left|\delta\left(v_{n}, w_{n}\right)\right|=0, \lim _{n \longrightarrow \infty} v_{n}=z .
$$


By triangular property of $(V, \delta)$,

$$
\begin{aligned}
\delta\left(w_{n}, z\right) & \leq b\left[\delta\left(w_{n}, v_{n}\right)+\delta\left(v_{n}, z\right)\right] \Rightarrow\left|\delta\left(w_{n}, z\right)\right| \\
& \leq b\left[\left|\delta\left(w_{n}, v_{n}\right)\right|+\left|\delta\left(v_{n}, z\right)\right|\right] .
\end{aligned}
$$

Now by applying $\lim _{n \longrightarrow \infty}$ and using (2), we have

$\lim _{n \rightarrow \infty}\left|\delta\left(w_{n}, z\right)\right| \leq \lim _{n \longrightarrow \infty} b\left|\delta\left(w_{n}, v_{n}\right)\right|+\lim _{n \rightarrow \infty} b\left|\delta\left(v_{n}, z\right)\right|=0+0$

Hence, we proved that $\lim _{n \rightarrow \infty} w_{n}=z$.

Definition 8 (see [27]). Let $(V, \delta)$ be a complex-valued bmetric space. A pair $(J, K)$ is said to be compatible iff $\lim _{n \rightarrow \infty}\left|\delta\left(J K v_{n}, K J v_{n}\right)\right|=0$, whenever $\left\{v_{n}\right\}$ is a sequence in $V$ such that

$$
\lim _{n \longrightarrow \infty} J v_{n}=\lim _{n \longrightarrow \infty} K v_{n}=z \text { for some } z \in V .
$$

\section{Main Result}

Theorem 9. Let $(V, \delta)$ be a complete complex-valued b-metric space and let $J, K, f: V \longrightarrow V$ be three self-mappings satisfying the following:

$$
\begin{aligned}
\delta\left(J v_{1}, K v_{2}\right) \leq & \eta_{1} \delta\left(f v_{1}, f v_{2}\right)+\eta_{2} \frac{\delta\left(f v_{1}, J v_{1}\right) \delta\left(f v_{2}, K v_{2}\right)}{1+\delta\left(f v_{1}, f v_{2}\right)} \\
& +\eta_{3} \frac{\left[\delta\left(f v_{1}, J v_{1}\right) \delta\left(f v_{1}, K v_{2}\right)+\delta\left(f v_{2}, K v_{2}\right) \delta\left(f v_{2}, J v_{1}\right)\right]}{\delta\left(f v_{1}, K v_{2}\right)+\delta\left(f v_{2}, J v_{1}\right)} \\
& +\eta_{4} \max \left\{\delta\left(f v_{1}, J v_{1}\right), \delta\left(f v_{2}, K v_{2}\right), \delta\right. \\
& \left.\cdot\left(f v_{1}, K v_{2}\right), \delta\left(f v_{2}, J v_{1}\right)\right\}
\end{aligned}
$$

for all $v_{1}, v_{2} \in V$, and $\eta_{1}, \eta_{2}, \eta_{3}, \eta_{4} \in[0,1)$ with $\left(\eta_{1}+\eta_{2}+\eta_{3}\right.$ $\left.+2 b \eta_{4}\right)<1$, where $b \geq 1$. If $f$ is continuous and $(f, J),(f, K)$ are compatible, then $f, J$ and $K$ have a unique common fixed point in $V$.

Proof. Fix $v_{0} \in V$, and we define some sequences in $V$ such that

$$
\hbar_{2 n}=f v_{2 n+1}=J v_{2 n}, \hbar_{2 n+1}=f v_{2 n+2}=K v_{2 n+1}, \forall n \geq 0
$$

Now by using (6),

$$
\begin{aligned}
\delta\left(\hbar_{2 n}, \hbar_{2 n+1}\right)= & \delta\left(J v_{2 n}, K v_{2 n+1}\right) \leq \eta_{1} \delta\left(f v_{2 n}, f v_{2 n+1}\right) \\
& +\eta_{2} \frac{\delta\left(f v_{2 n}, J v_{2 n}\right) \delta\left(f v_{2 n+1}, K v_{2 n+1}\right)}{1+\delta\left(f v_{2 n}, f v_{2 n+1}\right)} \\
& +\eta_{3} \frac{\left[\delta\left(f v_{2 n}, J v_{2 n}\right) \delta\left(f v_{2 n}, K v_{2 n+1}\right)+\delta\left(f v_{2 n+1}, K v_{2 n+1}\right) \delta\left(f v_{2 n+1}, J v_{2 n}\right)\right]}{\delta\left(f v_{2 n}, K v_{2 n+1}\right)+\delta\left(f v_{2 n+1}, J v_{2 n}\right)} \\
& +\eta_{4} \max \left\{\delta\left(f v_{2 n}, J v_{2 n}\right), \delta\left(f v_{2 n+1}, K v_{2 n+1}\right), \delta\right. \\
& \left.+\left(f v_{2 n}, K v_{2 n+1}\right), \delta\left(f v_{2 n+1}, J v_{2 n}\right)\right\} \\
= & \eta_{1} \delta\left(\hbar_{2 n-1}, \hbar_{2 n}\right)+\eta_{2} \frac{\delta\left(\hbar_{2 n-1}, \hbar_{2 n}\right) \delta\left(\hbar_{2 n}, \hbar_{2 n+1}\right)}{1+\delta\left(\hbar_{2 n-1}, \hbar_{2 n}\right)} \\
& +\eta_{3} \frac{\left[\delta\left(\hbar_{2 n-1}, \hbar_{2 n}\right) \delta\left(\hbar_{2 n-1}, \hbar_{2 n+1}\right)+\delta\left(\hbar_{2 n}, \hbar_{2 n+1}\right) \delta\left(\hbar_{2 n}, \hbar_{2 n}\right)\right]}{\delta\left(\hbar_{2 n-1}, \hbar_{2 n+1}\right)+\delta\left(\hbar_{2 n}, \hbar_{2 n}\right)} \\
& +\eta_{4} \max \left\{\delta\left(\hbar_{2 n-1}, \hbar_{2 n}\right), \delta\left(\hbar_{2 n}, \hbar_{2 n+1}\right), \delta\left(\hbar_{2 n-1}, \hbar_{2 n+1}\right), \delta\left(\hbar_{2 n}, \hbar_{2 n}\right)\right\} .
\end{aligned}
$$

This implies that

$$
\begin{aligned}
\left|\delta\left(\hbar_{2 n}, \hbar_{2 n+1}\right)\right| \leq & \eta_{1}\left|\delta\left(\hbar_{2 n-1}, \hbar_{2 n}\right)\right|+\eta_{2} \frac{\left|\delta\left(\hbar_{2 n-1}, \hbar_{2 n}\right)\right|\left|\delta\left(\hbar_{2 n}, \hbar_{2 n+1}\right)\right|}{\left|1+\delta\left(\hbar_{2 n-1}, \hbar_{2 n}\right)\right|} \\
& +\eta_{3} \frac{\left[\delta\left(\hbar_{2 n-1}, \hbar_{2 n}\right)|| \delta\left(\hbar_{2 n-1}, \hbar_{2 n+1}\right)|+| \delta\left(\hbar_{2 n}, \hbar_{2 n+1}\right)|| \delta\left(\hbar_{2 n}, \hbar_{2 n}\right) \mid\right]}{\left|\delta\left(\hbar_{2 n-1}, \hbar_{2 n+1}\right)\right|+\left|\delta\left(\hbar_{2 n}, \hbar_{2 n}\right)\right|} \\
& +\eta_{4} \max \left\{\left|\delta\left(\hbar_{2 n-1}, \hbar_{2 n}\right)\right|,\left|\delta\left(\hbar_{2 n}, \hbar_{2 n+1}\right)\right|,\left|\delta\left(\hbar_{2 n-1}, \hbar_{2 n+1}\right)\right|,\right. \\
& \left.\cdot\left|\delta\left(\hbar_{2 n}, \hbar_{2 n}\right)\right|\right\} .
\end{aligned}
$$

After simplification, we get that

$$
\begin{aligned}
\left|\delta\left(\hbar_{2 n}, \hbar_{2 n+1}\right)\right| \leq & \left(\eta_{1}+\eta_{3}\right)\left|\delta\left(\hbar_{2 n-1}, \hbar_{2 n}\right)\right|+\eta_{2}\left|\delta\left(\hbar_{2 n}, \hbar_{2 n+1}\right)\right| \\
& +\eta_{4} \max \left\{\left|\delta\left(\hbar_{2 n-1}, \hbar_{2 n}\right)\right|,\left|\delta\left(\hbar_{2 n}, \hbar_{2 n+1}\right)\right|,\right. \\
& \left.\cdot\left|\delta\left(\hbar_{2 n-1}, \hbar_{2 n+1}\right)\right|\right\} .
\end{aligned}
$$

Now there are three possibilities:

(i) If $\delta\left(\hbar_{2 n-1}, \hbar_{2 n}\right)$ is a maximum term in $\left\{\mid \delta\left(\hbar_{2 n-1}, \hbar_{2 n}\right)\right.$ |, $\left.\left|\delta\left(\hbar_{2 n}, \hbar_{2 n+1}\right)\right|,\left|\delta\left(\hbar_{2 n-1}, \hbar_{2 n+1}\right)\right|\right\}$, then after simplification, (10) can be written as follows:

$\left|\delta\left(\hbar_{2 n}, \hbar_{2 n+1}\right)\right| \leq a_{1}\left|\delta\left(\hbar_{2 n-1}, \hbar_{2 n}\right)\right|$, where $a_{1}=\frac{\eta_{1}+\eta_{3}+\eta_{4}}{1-\eta_{2}}<1$

(ii) If $\delta\left(\hbar_{2 n}, \hbar_{2 n+1}\right)$ is a maximum term in $\left\{\mid \delta\left(\hbar_{2 n-1}, \hbar_{2 n}\right)\right.$ $\left.|,| \delta\left(\hbar_{2 n}, \hbar_{2 n+1}\right)|,| \delta\left(\hbar_{2 n-1}, \hbar_{2 n+1}\right) \mid\right\}$, then after simplification, (10) can be written as follows:

$\left|\delta\left(\hbar_{2 n}, \hbar_{2 n+1}\right)\right| \leq a_{2}\left|\delta\left(\hbar_{2 n-1}, \hbar_{2 n}\right)\right|$, where $a_{2}=\frac{\eta_{1}+\eta_{3}}{1-\eta_{2}-\eta_{4}}<1$

(iii) If $\delta\left(\hbar_{2 n-1}, \hbar_{2 n+1}\right)$ is a maximum term in $\left\{\mid \delta\left(\hbar_{2 n-1}\right.\right.$, $\left.\left.\hbar_{2 n}\right)|,| \delta\left(\hbar_{2 n}, \hbar_{2 n+1}\right)|,| \delta\left(\hbar_{2 n-1}, \hbar_{2 n+1}\right) \mid\right\}$, and by using the triangular property of complex-valued b-metric 
space, then after simplification, (10) can be written as follows:

$\left|\delta\left(\hbar_{2 n}, \hbar_{2 n+1}\right)\right| \leq a_{3}\left|\delta\left(\hbar_{2 n-1}, \hbar_{2 n}\right)\right|$, where $a_{3}=\frac{\eta_{1}+\eta_{3}+\eta_{4} b}{1-\eta_{2}-\eta_{4} b}<1$.

Let $a:=\max \left\{a_{1}, a_{2}, a_{3}\right\}<1$; then, from (11), (12), and (13), for all $n \geq 0$, we have

$$
\left|\delta\left(\hbar_{2 n}, \hbar_{2 n+1}\right)\right| \leq a\left|\delta\left(\hbar_{2 n-1}, \hbar_{2 n}\right)\right|
$$

Similarly,

$$
\left|\delta\left(\hbar_{2 n-1}, \hbar_{2 n}\right)\right| \leq a\left|\delta\left(\hbar_{2 n-2}, \hbar_{2 n-1}\right)\right| .
$$

Now from (15) and (14), and by induction, we have that,

$$
\begin{aligned}
\left|\delta\left(\hbar_{2 n}, \hbar_{2 n+1}\right)\right| & \leq a\left|\delta\left(\hbar_{2 n-1}, \hbar_{2 n}\right)\right| \leq a^{2}\left|\delta\left(\hbar_{2 n-2}, \hbar_{2 n-1}\right)\right| \\
& \leq \cdots \leq a^{2 n}\left|\delta\left(\hbar_{0}, \hbar_{1}\right)\right| .
\end{aligned}
$$

Next, we show that $\left\{\hbar_{n}\right\}$ is a Cauchy sequence. Let $m, n$ $\in \mathbb{N}$ and $m>n$, then we have

$$
\begin{aligned}
\left|\delta\left(\hbar_{n}, \hbar_{m}\right)\right| & \leq b\left|\delta\left(\hbar_{n}, \hbar_{n+1}\right)\right|+b\left|\delta\left(\hbar_{n+1}, \hbar_{m}\right)\right| \\
& \leq b\left|\delta\left(\hbar_{n}, \hbar_{n+1}\right)\right|+b^{2}\left|\delta\left(\hbar_{n+1}, \hbar_{n+2}\right)\right|+\cdots+b^{m-n}\left|\delta\left(\hbar_{m-1}, \hbar_{m}\right)\right| \\
& \leq b a^{n}\left|\delta\left(\hbar_{0}, \hbar_{1}\right)\right|+b^{2} a^{n+1}\left|\delta\left(\hbar_{0}, \hbar_{1}\right)\right|+\cdots+b^{m-n} a^{m-1}\left|\delta\left(\hbar_{0}, \hbar_{1}\right)\right| \\
& \leq\left[b a^{n}+b^{2} a^{n+1}+\cdots+b^{m-n} a^{m-1}\right]\left|\delta\left(\hbar_{0}, \hbar_{1}\right)\right| \\
& =\left[b a^{n}+b^{2} a^{n+1}+\cdots+b^{m-n} a^{m-1}\right]\left|\delta\left(\hbar_{0}, \hbar_{1}\right)\right| \\
& =b a^{n}\left[1+b a+b^{2} a^{2} \cdots+b^{m-(n+1)} a^{m-(n+1)}\right]\left|\delta\left(\hbar_{0}, \hbar_{1}\right)\right| \\
& =b a^{n} \sum_{t=0}^{m-(n+1)} b^{t} a^{t}\left|\delta\left(\hbar_{0}, \hbar_{1}\right)\right| \leq b a^{n} \sum_{t=0}^{\infty} b^{t} a^{t}\left|\delta\left(\hbar_{0}, \hbar_{1}\right)\right| \\
& =\frac{b a^{n}}{1-b a}\left|\delta\left(\hbar_{0}, \hbar_{1}\right)\right| \longrightarrow 0, \text { as } n \longrightarrow \infty .
\end{aligned}
$$

Hence, $\left\{\hbar_{n}\right\}$ is a Cauchy sequence. Since $V$ is a complete complex-valued b-metric space, there exists $p \in V$ such that $\hbar_{n} \longrightarrow p$, as $n \longrightarrow \infty$ or $\lim \hbar_{n}=p$, and from (7), we have

$$
\lim _{n \longrightarrow \infty} f v_{2 n+1}=p, \lim _{n \longrightarrow \infty} J v_{2 n}=p, \lim _{n \longrightarrow \infty} K v_{2 n+1}=p .
$$

Since $f$ is a continuous self-map on $V$, therefore

$\lim _{n \longrightarrow \infty} f\left(f v_{2 n+1}\right)=f p, \lim _{n \longrightarrow \infty} f\left(J v_{2 n}\right)=f p, \lim _{n \longrightarrow \infty} f\left(K v_{2 n+1}\right)=f p$

As a pair $(f, J)$ is compatible, so for some sequence $\left\{v_{2 n}\right\}$ in $V$ and by the definition of compatibility, we have that

$$
\lim _{n \rightarrow \infty}\left|\delta\left(J\left(f v_{2 n}\right), f\left(J v_{2 n}\right)\right)\right|=0 .
$$

Now from (19), (20), and by using Lemma 7, we have

$$
\lim _{n \rightarrow \infty} J\left(f v_{2 n}\right)=f p
$$

Next, we have to show that $f p=p$, so by putting $v_{1}=f v_{2 n}$ and $v_{2}=v_{2 n+1}$, in (6),

$$
\begin{aligned}
& \delta\left(J\left(f v_{2 n}\right), K v_{2 n+1}\right) \leq \eta_{1} \delta\left(f\left(f v_{2 n}\right), f v_{2 n+1}\right)+\eta_{2} \frac{\delta\left(f\left(f v_{2 n}\right), J\left(f v_{2 n}\right)\right) \delta\left(f v_{2 n+1}, K v_{2 n+1}\right)}{1+\delta\left(f\left(f v_{2 n}\right), f v_{2 n+1}\right)} \\
& \quad+\eta_{3} \frac{\left[\delta\left(f\left(f v_{2 n}\right), J\left(f v_{2 n}\right)\right) \delta\left(f\left(f v_{2 n}\right), K v_{2 n+1}\right)+\delta\left(f v_{2 n+1}, K v_{2 n+1}\right) \delta\left(f v_{2 n+1}, J\left(f v_{2 n}\right)\right)\right]}{\delta\left(f\left(f v_{2 n}\right), K v_{2 n+1}\right)+\delta\left(f v_{2 n+1}, J\left(f v_{2 n}\right)\right)} \\
& \quad+\eta_{4} \max \left\{\delta\left(f\left(f v_{2 n}\right), J\left(f v_{2 n}\right)\right), \delta\left(f v_{2 n+1}, K v_{2 n+1}\right), \delta\right. \\
& \left.\left.\quad \cdot\left(f\left(f v_{2 n}\right), K v_{2 n+1}\right)\right), \delta\left(f v_{2 n+1}, J\left(f v_{2 n}\right)\right)\right\} .
\end{aligned}
$$

This implies that

$$
\begin{aligned}
& \left|\delta\left(J\left(f v_{2 n}\right), K v_{2 n+1}\right)\right| \leq \eta_{1}\left|\delta\left(f\left(f v_{2 n}\right), f v_{2 n+1}\right)\right|+\eta_{2} \frac{\left|\delta\left(f\left(f v_{2 n}\right), J\left(f v_{2 n}\right)\right)\right|\left|\delta\left(f v_{2 n+1}, K v_{2 n+1}\right)\right|}{\left|1+\delta\left(f\left(f v_{2 n}\right), f v_{2 n+1}\right)\right|} \\
& \quad+\eta_{3} \frac{\left[\delta\left(f\left(f v_{2 n}\right), J\left(f v_{2 n}\right)\right)|| \delta\left(f\left(f v_{2 n}\right), K v_{2 n+1}\right)|+| \delta\left(f v_{2 n+1}, K v_{2 n+1}\right)|| \delta\left(f v_{2 n+1}, J\left(f v_{2 n}\right)\right) \mid\right]}{\left|\delta\left(f\left(f v_{2 n}\right), K v_{2 n+1}\right)\right|+\left|\delta\left(f v_{2 n+1}, J\left(f v_{2 n}\right)\right)\right|} \\
& \quad+\eta_{4} \max \left\{\left|\delta\left(f\left(f v_{2 n}\right), J\left(f v_{2 n}\right)\right)\right|,\left|\delta\left(f v_{2 n+1}, K v_{2 n+1}\right)\right|,\right. \\
& \left.\quad \cdot\left|\delta\left(f\left(f v_{2 n}\right), K v_{2 n+1}\right)\right|,\left|\delta\left(f v_{2 n+1}, J\left(f v_{2 n}\right)\right)\right|\right\} .
\end{aligned}
$$

Now applying $\lim _{n \rightarrow \infty}$ on both sides and from (18), (19), and (21), we get that

$$
\begin{aligned}
|\delta(f p, p)| \leq & \eta_{1}|\delta(f p, p)|+\eta_{2} \frac{|\delta(f p, f p)||\delta(p, p)|}{|1+\delta(f p, p)|} \\
& +\eta_{3} \frac{[|\delta(f p, f p)||\delta(f p, p)|+|\delta(p, p)||\delta(p, f p)|]}{|\delta(f p, p)|+|\delta(p, f p)|} \\
& +\eta_{4} \max \{|\delta(f p, f p)|,|\delta(p, p)|,|\delta(f p, p)|,|\delta(p, f p)|\} .
\end{aligned}
$$

After simplification, we get that

$|\delta(f p, p)| \leq\left(\eta_{1}+\eta_{4}\right)|\delta(f p, p)| \Rightarrow\left(1-\eta_{1}-\eta_{4}\right)|\delta(f p, p)| \leq 0$.

Since $\left(1-\eta_{1}-\eta_{4}\right) \neq 0 \Rightarrow|\delta(f p, p)|=0$, hence we get that

$$
f p=p
$$

Next, we have to show that $J p=p$, and by the view of (6),

$$
\begin{aligned}
\delta\left(J p, f v_{2 n+2}\right)= & \delta\left(J p, K v_{2 n+1}\right) \leq \eta_{1} \delta\left(f p, f v_{2 n+1}\right)+\eta_{2} \frac{\delta(f p, J p) \delta\left(f v_{2 n+1}, K v_{2 n+1}\right)}{1+\delta\left(f p, f v_{2 n+1}\right)} \\
& +\eta_{3} \frac{\left[\delta(f p, J p) \delta\left(f p, K v_{2 n+1}\right)+\delta\left(f v_{2 n+1}, K v_{2 n+1}\right) \delta\left(f v_{2 n+1}, J p\right)\right]}{\delta\left(f p, K v_{2 n+1}\right)+\delta\left(f v_{2 n+1}, J p\right)} \\
& +\eta_{4} \max \left\{\delta(f p, J p), \delta\left(f v_{2 n+1}, K v_{2 n+1}\right), \delta\left(f p, K v_{2 n+1}\right), \delta\right. \\
& \left.\cdot\left(f v_{2 n+1}, J p\right)\right\} .
\end{aligned}
$$

This implies that 


$$
\begin{aligned}
\left|\delta\left(J p, f v_{2 n+2}\right)\right| \leq & \eta_{1}\left|\delta\left(f p, f v_{2 n+1}\right)\right|+\eta_{2} \frac{|\delta(f p, J p)|\left|\delta\left(f v_{2 n+1}, K v_{2 n+1}\right)\right|}{\left|1+\delta\left(f p, f v_{2 n+1}\right)\right|} \\
& +\eta_{3} \frac{\left[\delta(f p, J p)|| \delta\left(f p, K v_{2 n+1}\right)|+| \delta\left(f v_{2 n+1}, K v_{2 n+1}\right)|| \delta\left(f v_{2 n+1}, J p\right) \mid\right]}{\left|\delta\left(f p, K v_{2 n+1}\right)\right|+\left|\delta\left(f v_{2 n+1}, J p\right)\right|} \\
& +\eta_{4} \max \left\{|\delta(f p, J p)|,\left|\delta\left(f v_{2 n+1}, K v_{2 n+1}\right)\right|,\left|\delta\left(f p, K v_{2 n+1}\right)\right|,\right. \\
& \left.\cdot\left|\delta\left(f v_{2 n+1}, J p\right)\right|\right\} .
\end{aligned}
$$

Now again applying $\lim _{n \rightarrow \infty}$ on both sides and by using (18) and (26), we have that

$$
\begin{aligned}
|\delta(J p, p)| \leq & \eta_{1}|\delta(f p, p)|+\eta_{2} \frac{|\delta(f p, J p)||\delta(p, p)|}{|1+\delta(f p, p)|} \\
& +\eta_{3} \frac{[|\delta(f p, J p)||\delta(f p, p)|+|\delta(p, p)||\delta(p, J p)|]}{|\delta(f p, p)|+|\delta(p, J p)|}+\eta_{4} \max \\
& \cdot\{|\delta(f p, J p)|,|\delta(p, p)|,|\delta(f p, p)|,|\delta(p, J p)|\}=\eta_{4}|\delta(p, J p)| .
\end{aligned}
$$

This implies that $\left(1-\eta_{4}\right)|\delta(J p, p)| \leq 0$. Since $\left(1-\eta_{4}\right) \neq$ $0 \Rightarrow|\delta(J p, p)|=0$, hence

$$
J p=p
$$

Now, we have to show that $K p=p$, and by using (6),

$$
\begin{aligned}
\delta\left(f v_{2 n+1}, K p\right)= & \delta\left(J v_{2 n}, K p\right) \leq \eta_{1} \delta\left(f v_{2 n}, f p\right)+\eta_{2} \frac{\delta\left(f v_{2 n}, J v_{2 n}\right) \delta(f p, K p)}{1+\delta\left(f v_{2 n}, f p\right)} \\
& +\eta_{3} \frac{\left[\delta\left(f v_{2 n}, J v_{2 n}\right) \delta\left(f v_{2 n}, K p\right)+\delta(f p, K p) \delta\left(f p, J v_{2 n}\right)\right]}{\delta\left(f v_{2 n}, K p\right)+\delta\left(f p, J v_{2 n}\right)} \\
& +\eta_{4} \max \left\{\delta\left(f v_{2 n}, J v_{2 n}\right), \delta(f p, K p), \delta\left(f v_{2 n}, K p\right), \delta\right. \\
& \left.\cdot\left(f p, J v_{2 n}\right)\right\} .
\end{aligned}
$$

This implies that

$$
\begin{aligned}
\left|\delta\left(f v_{2 n+1}, K p\right)\right| \leq & \eta_{1}\left|\delta\left(f v_{2 n}, f p\right)\right|+\eta_{2} \frac{\left|\delta\left(f v_{2 n}, J v_{2 n}\right)\right||\delta(f p, K p)|}{\left|1+\delta\left(f v_{2 n}, f p\right)\right|} \\
& +\eta_{3} \frac{\left[\delta\left(f v_{2 n}, J v_{2 n}\right)|| \delta\left(f v_{2 n}, K p\right)|+| \delta(f p, K p)|| \delta\left(f p, J v_{2 n}\right) \mid\right]}{\left|\delta\left(f v_{2 n}, K p\right)\right|+\left|\delta\left(f p, J v_{2 n}\right)\right|} \\
& +\eta_{4} \max \left\{\left|\delta\left(f v_{2 n}, J v_{2 n}\right)\right|,|\delta(f p, K p)|,\left|\delta\left(f v_{2 n}, K p\right)\right|\right. \\
& \left.\cdot\left|\delta\left(f p, J v_{2 n}\right)\right|\right\} .
\end{aligned}
$$

Applying $\lim _{n \longrightarrow \infty}$ on both sides and by using (18) and (26), we have that

$$
\begin{aligned}
|\delta(p, K p)| \leq & \eta_{1}|\delta(p, f p)|+\eta_{2} \frac{|\delta(p, p)||\delta(f p, K p)|}{|1+\delta(p, f p)|} \\
& +\eta_{3} \frac{[|\delta(p, p)||\delta(p, K p)|+|\delta(f p, K p)||\delta(f p, p)|]}{|\delta(p, K p)|+|\delta(f p, p)|}+\eta_{4} \max \\
& \cdot\{|\delta(p, p)|,|\delta(f p, K p)|,|\delta(p, K p)|,|\delta(f p, p)|\}=\eta_{4}|\delta(p, K p)| .
\end{aligned}
$$

This implies that $\left(1-\eta_{4}\right)|\delta(p, K p)| \leq 0$. Since $\left(1-\eta_{4}\right) \neq$ $0 \Rightarrow|\delta(p, K p)|=0$, hence

$$
K p=p
$$

Now from (26), (30), and (34), we get that $p$ is a common fixed point of $f, J$ and $K$, i.e.,

$$
f p=J p=K p=p
$$

Uniqueness: assume that $p^{*} \in V$ is an other common fixed point of $f, J$ and $K$ along with $p$, i.e.,

$$
\begin{gathered}
f p=J p=K p=p, \\
f p^{*}=J p^{*}=K p^{*}=p^{*}
\end{gathered}
$$

Then, from (6), we have that

$$
\begin{aligned}
\delta\left(p, p^{*}\right)= & \delta\left(J p, K p^{*}\right) \leq \eta_{1} \delta\left(f p, f p^{*}\right)+\eta_{2} \frac{\delta(f p, J p) \delta\left(f p^{*}, K p^{*}\right)}{1+\delta\left(f p, f p^{*}\right)} \\
& +\eta_{3} \frac{\left[\delta(f p, J p) \delta\left(f p, K p^{*}\right)+\delta\left(f p^{*}, K p^{*}\right) \delta\left(f p^{*}, J p\right)\right]}{\delta\left(f p, K p^{*}\right)+\delta\left(f p^{*}, J p\right)}+\eta_{4} \max \\
& \cdot\left\{\delta(f p, J p), \delta\left(f p^{*}, K p^{*}\right), \delta\left(f p, K p^{*}\right), \delta\left(f p^{*}, J p\right)\right\}=\left(\eta_{1}+\eta_{4}\right) \delta\left(p, p^{*}\right) .
\end{aligned}
$$

This implies that $\left|\delta\left(p, p^{*}\right)\right| \leq\left(\eta_{1}+\eta_{4}\right)\left|\delta\left(p, p^{*}\right)\right| \Rightarrow(1-$ $\left.\eta_{1}-\eta_{4}\right)\left|\delta\left(p, p^{*}\right)\right| \leq 0$, since $\left(1-\eta_{1}-\eta_{4}\right) \neq 0 \Rightarrow\left|\delta\left(p, p^{*}\right)\right|=0$ $\Rightarrow p=p^{*}$. Hence, we proved that $f, J$ and $K$ have a unique common fixed point in $V$.

Remark 10.

(i) If we put $\eta_{1}=\lambda, \eta_{2}=\mu$, and $\eta_{3}=\eta_{4}=0$ in Theorem 9, we get the results of [26] Theorem 15 .

(ii) If we put $\eta_{2}=a$ and $\eta_{1}=\eta_{3}=\eta_{4}=0$ in Theorem 9, we get the results of [26] Theorem 19.

Example 2. Let $(V, \delta)$ be a complex-valued b-metric space, where $V=[0,1)$ and $\delta: V \times V \longrightarrow \mathbb{C}$ with $\delta\left(v_{1}, v_{2}\right)=4$ $\left|v_{1}-v_{2}\right|^{2} / 9+i\left(4\left|v_{1}-v_{2}\right|^{2} / 9\right)$, for all $v_{1}, v_{2} \in V$. Now to find the value of $b$, we have that

$$
\begin{aligned}
\delta\left(v_{1}, v_{2}\right)= & \frac{4\left|v_{1}-v_{2}\right|^{2}}{9}+i \frac{4\left|v_{1}-v_{2}\right|^{2}}{9} \\
= & \frac{4\left|\left(v_{1}-v_{3}\right)+\left(v_{3}-v_{2}\right)\right|^{2}}{9}+i \frac{4\left|\left(v_{1}-v_{3}\right)+\left(v_{3}-v_{2}\right)\right|^{2}}{9} \\
\leq & {\left[\frac{4\left|v_{1}-v_{3}\right|^{2}}{9}+\frac{4\left|v_{3}-v_{2}\right|^{2}}{9}+\frac{4}{9}\left(2\left|v_{1}-v_{3}\right|\left|v_{3}-v_{2}\right|\right)\right] } \\
& +i\left[\frac{4\left|v_{1}-v_{3}\right|^{2}}{9}+\frac{4\left|v_{3}-v_{2}\right|^{2}}{9}+\frac{4}{9}\left(2\left|v_{1}-v_{3}\right|\left|v_{3}-v_{2}\right|\right)\right] \\
\leq & {\left[\frac{4\left|v_{1}-v_{3}\right|^{2}}{9}+\frac{4\left|v_{3}-v_{2}\right|^{2}}{9}+\frac{4\left|v_{1}-v_{3}\right|^{2}}{9}+\frac{4\left|v_{3}-v_{2}\right|^{2}}{9}\right] } \\
& +i\left[\frac{4\left|v_{1}-v_{3}\right|^{2}}{9}+\frac{4\left|v_{3}-v_{2}\right|^{2}}{9}+\frac{4\left|v_{1}-v_{3}\right|^{2}}{9}+\frac{4\left|v_{3}-v_{2}\right|^{2}}{9}\right] \\
= & 2\left[\frac{4\left|v_{1}-v_{3}\right|^{2}}{9}+\frac{4\left|v_{3}-v_{2}\right|^{2}}{9}\right]+i 2\left[\frac{4\left|v_{1}-v_{3}\right|^{2}}{9}+\frac{4\left|v_{3}-v_{2}\right|^{2}}{9}\right] \\
= & 2\left[\frac{4\left|v_{1}-v_{3}\right|^{2}}{9}+i \frac{4\left|v_{1}-v_{3}\right|^{2}}{9}+\frac{4\left|v_{3}-v_{2}\right|^{2}}{9}+i \frac{4\left|v_{3}-v_{2}\right|^{2}}{9}\right] \\
= & 2\left[\delta\left(v_{1}, v_{3}\right)+\delta\left(v_{3}, v_{2}\right)\right] .
\end{aligned}
$$


That is, $\delta\left(v_{1}, v_{2}\right) \leq b\left[\delta\left(v_{1}, v_{3}\right)+\delta\left(v_{3}, v_{2}\right)\right]$ with $b=2$. Now we define $J, K, f: V \longrightarrow V$ as

$$
J v_{1}=K v_{1}=\frac{2 v_{1}}{35} \text { and } f v_{1}=\frac{v_{1}}{5} \text {. }
$$

Notice that

$$
\left\{\begin{array}{c}
\left|\delta\left(f v_{1}, f v_{2}\right)\right|, \frac{\left|\delta\left(f v_{1}, J v_{1}\right)\right|\left|\delta\left(f v_{2}, K v_{2}\right)\right|}{\left|1+\delta\left(f v_{1}, f v_{2}\right)\right|}, \frac{\left[\left|\delta\left(f v_{1}, J v_{1}\right)\right|\left|\delta\left(f v_{1}, K v_{2}\right)\right|+\left|\delta\left(f v_{2}, K v_{2}\right)\right|\left|\delta\left(f v_{2}, J v_{1}\right)\right|\right]}{\left|\delta\left(f v_{1}, K v_{2}\right)\right|+\left|\delta\left(f v_{2}, J v_{1}\right)\right|}, \\
\max \left\{\left|\delta\left(f v_{1}, J v_{1}\right)\right|,\left|\delta\left(f v_{2}, K v_{2}\right)\right|,\left|\delta\left(f v_{1}, K v_{2}\right)\right|,\left|\delta\left(f v_{2}, J v_{1}\right)\right|\right\}
\end{array}\right\} \geq 0
$$

In all regards, it is enough to show that $\delta\left(J v_{1}, K v_{2}\right) \leq \eta_{1}$ $\delta\left(f v_{1}, f v_{2}\right)$, for all $v_{1}, v_{2} \in[0,1]$ and $\eta_{1}, \eta_{2}, \eta_{3}, \eta_{4} \in[0,1)$, with $\left(\eta_{1}+\eta_{2}+\eta_{3}+2 \eta_{4} b\right)<1$.

$$
\begin{aligned}
\delta\left(J v_{1}, K v_{2}\right) & =\left[\frac{4\left|J v_{1}-K v_{2}\right|^{2}}{9}+i \frac{4\left|J v_{1}-K v_{2}\right|^{2}}{9}\right] \\
& =\left[\frac{4\left|2 v_{1} / 35-2 v_{2} / 35\right|^{2}}{9}+i \frac{4\left|2 v_{1} / 35-2 v_{2} / 35\right|^{2}}{9}\right] \\
& =\left(\frac{2}{7}\right)^{2}\left[\frac{4\left|v_{1} / 5-v_{2} / 5\right|^{2}}{9}+i \frac{4\left|v_{1} / 5-v_{2} / 5\right|^{2}}{9}\right] \\
& =\frac{4}{49}\left[\frac{4\left|v_{1} / 5-v_{2} / 5\right|^{2}}{9}+i \frac{4\left|v_{1} / 5-v_{2} / 5\right|^{2}}{9}\right] .
\end{aligned}
$$

And

$$
\begin{aligned}
\delta\left(f v_{1}, f v_{2}\right) & =\left[\frac{4\left|f v_{1}-f v_{2}\right|^{2}}{9}+i \frac{4\left|f v_{1}-f v_{2}\right|^{2}}{9}\right] \\
& =\left[\frac{4\left|v_{1} / 5-v_{2} / 5\right|^{2}}{9}+i \frac{4\left|v_{1} / 5-v_{2} / 5\right|^{2}}{9}\right] .
\end{aligned}
$$

For $v_{1}, v_{2} \in[0,1]$, we discuss different cases with $\eta_{1}=2 /$ $5, \eta_{2}=1 / 5, \eta_{3}=1 / 10, \eta_{4}=1 / 20$, and $b=2$. Hence,

$$
\eta_{1}+\eta_{2}+\eta_{3}+2 \eta_{4} b=\frac{2}{5}+\frac{1}{5}+\frac{1}{10}+2\left(\frac{1}{20}\right) 2<1 .
$$

Case 1. Let $v_{1}=0, v_{2}=0$; then, from (41) and (42), directly we get that $\delta\left(J v_{1}, K v_{2}\right) \leq \eta_{1} \delta\left(f v_{1}, f v_{2}\right)$. Hence, (6) is satisfied with $\eta_{1}=2 / 5, \eta_{2}=1 / 5, \eta_{3}=1 / 10, \eta_{4}=1 / 20$, and $b=2$.

Case 2. Let $v_{1}=1, v_{2}=0$; then, from (41) and (42), we find $\delta\left(J v_{1}, K v_{2}\right) \leq \eta_{1} \delta\left(f v_{1}, f v_{2}\right)$ is satisfied with $\eta_{1}=2 / 5$, i.e,

$$
\begin{aligned}
\frac{4}{49} & {\left[\frac{4|1 / 5-0 / 5|^{2}}{9}+i \frac{4|1 / 5-0 / 5|^{2}}{9}\right] } \\
& \leq \eta_{1}\left[\frac{4|1 / 5-0 / 5|^{2}}{9}+i \frac{4|1 / 5-0 / 5|^{2}}{9}\right] 0.0014[1+i] \\
& \leq 0.0071[1+i] .
\end{aligned}
$$

Hence, (6) is satisfied with $\eta_{1}=2 / 5, \eta_{2}=1 / 5, \eta_{3}=1 / 10$, $\eta_{4}=1 / 20$, and $b=2$.

Case 3. Let $v_{1}=1 / 2, v_{2}=1 / 4$; then from (41) and (42), we find $\delta\left(J v_{1}, K v_{2}\right) \leq \eta_{1} \delta\left(f v_{1}, f v_{2}\right)$ is satisfied with $\eta_{1}=2 / 5$, i.e,

$$
\begin{aligned}
\frac{4}{49}\left[\frac{4}{3600}+i \frac{4}{3600}\right] & \leq \eta_{1}\left[\frac{4}{3600}+i \frac{4}{3600}\right] 0.000090[1+i] \\
& \leq 0.00044[1+i] .
\end{aligned}
$$

Hence, (6) is satisfied with $\eta_{1}=2 / 5, \eta_{2}=1 / 5, \eta_{3}=1 / 10$, $\eta_{4}=1 / 20$, and $b=2$.

Case 4. Let $v_{1}=1, v_{2}=1$; then, from (41) and (42), directly we get that $\delta\left(J v_{1}, K v_{2}\right) \leq \eta_{1} \delta\left(f v_{1}, f v_{2}\right)$. Hence, (6) is satisfied with $\eta_{1}=2 / 5, \eta_{2}=1 / 5, \eta_{3}=1 / 10, \eta_{4}=1 / 20$, and $b=2$. Thus, all the conditions of Theorem 9 are satisfied with noticing that the point $0 \in V$, which remains fixed under mappings $f, J$ and $K$, is indeed unique.

Corollary 11. Let $(V, \delta)$ be a complete complex-valued $b$ metric space and let $J, K, f: V \longrightarrow V$ be three self-mappings satisfying the following:

$$
\begin{aligned}
\delta\left(J v_{1}, K v_{2}\right) \leq & \eta_{1} \delta\left(f v_{1}, f v_{2}\right)+\eta_{2} \frac{\delta\left(f v_{1}, J v_{1}\right) \delta\left(f v_{2}, K v_{2}\right)}{1+\delta\left(f v_{1}, f v_{2}\right)} \\
& +\eta_{3} \frac{\left[\delta\left(f v_{1}, J v_{1}\right) \delta\left(f v_{1}, K v_{2}\right)+\delta\left(f v_{2}, K v_{2}\right) \delta\left(f v_{2}, J v_{1}\right)\right]}{\delta\left(f v_{1}, K v_{2}\right)+\delta\left(f v_{2}, J v_{1}\right)} \\
& +\eta_{4}\left[\delta\left(f v_{1}, J v_{1}\right)+\delta\left(f v_{2}, K v_{2}\right)\right],
\end{aligned}
$$

for all $v_{1}, v_{2} \in V$ and $\eta_{1}, \eta_{2}, \eta_{3}, \eta_{4} \in[0,1)$ such that $\left(\eta_{1}+\eta_{2}\right.$ $\left.+\eta_{3}+2 \eta_{4}\right)<1$. If $f$ is a continuous self-mapping and $(f, J)$, $(f, K)$ are compatible, then $f, J$ and $K$ have a unique common fixed point in $V$.

Corollary 12. Let $(V, \delta)$ be a complete complex-valued $b$ metric space and let $J, K, f: V \longrightarrow V$ be three self-mappings satisfying the following: 


$$
\begin{aligned}
\delta\left(J v_{1}, K v_{2}\right) \leq & \eta_{1} \delta\left(f v_{1}, f v_{2}\right)+\eta_{2} \frac{\delta\left(f v_{1}, J v_{1}\right) \delta\left(f v_{2}, K v_{2}\right)}{1+\delta\left(f v_{1}, f v_{2}\right)} \\
& +\eta_{3} \frac{\left[\delta\left(f v_{1}, J v_{1}\right) \delta\left(f v_{1}, K v_{2}\right)+\delta\left(f v_{2}, K v_{2}\right) \delta\left(f v_{2}, J v_{1}\right)\right]}{\delta\left(f v_{1}, K v_{2}\right)+\delta\left(f v_{2}, J v_{1}\right)} \\
& +\eta_{4}\left[\delta\left(f v_{1}, K v_{2}\right)+\delta\left(f v_{2}, J v_{1}\right)\right]
\end{aligned}
$$

for all $v_{1}, v_{2} \in V$ and $\eta_{1}, \eta_{2}, \eta_{3}, \eta_{4} \in[0,1)$ such that $\left(\eta_{1}+\eta_{2}\right.$ $\left.+\eta_{3}+2 \eta_{4} b\right)<1$, where $b \geq 1$. If $f$ is continuous and $(f, J)$, $(f, K)$ are compatible, then $f, J$ and $K$ have a unique common fixed point in $V$.

Theorem 13. Let $(V, \delta)$ be a complete complex-valued $b$ metric space and let $J, K, f: V \longrightarrow V$ be three self-mappings satisfying:

$$
\begin{aligned}
\delta\left(J v_{1}, K v_{2}\right) \leq & \eta_{1} \delta\left(f v_{1}, f v_{2}\right)+\eta_{2} \frac{\delta\left(f v_{1}, J v_{1}\right) \delta\left(f v_{2}, K v_{2}\right)}{1+\delta\left(f v_{1}, f v_{2}\right)} \\
& +\eta_{3} \frac{\left[\delta\left(f v_{1}, J v_{1}\right) \delta\left(f v_{1}, K v_{2}\right)+\delta\left(f v_{2}, K v_{2}\right) \delta\left(f v_{2}, J v_{1}\right)\right]}{\delta\left(f v_{1}, K v_{2}\right)+\delta\left(f v_{2}, J v_{1}\right)} \\
& +\eta_{4}\left[\delta\left(f v_{1}, J v_{1}\right)+\delta\left(f v_{2}, K v_{2}\right)+\delta\left(f v_{1}, K v_{2}\right)+\delta\left(f v_{2}, J v_{1}\right)\right],
\end{aligned}
$$

for all $v_{1}, v_{2} \in V, \eta_{1}, \eta_{2}, \eta_{3} \in[0,1)$, and $\eta_{4} \in[0,1 / 4)$, such that $\left(\eta_{1}+\eta_{2}+\eta_{3}+2 \eta_{4}+2 \eta_{4} b\right)<1$, where $b \geq 1$. If $f$ is a continuous self-mapping and $(f, J),(f, K)$ are compatible, then $f, J$ and $K$ have a unique common fixed point in $V$.

Proof. Fix $v_{0} \in V$, and we define some sequences in $V$ such that

$$
\hbar_{2 n}=f v_{2 n+1}=J v_{2 n}, \hbar_{2 n+1}=f v_{2 n+2}=K v_{2 n+1} \text {, for all } n \geq 0
$$

Now by view of (48) and (49),

$$
\begin{aligned}
\delta\left(\hbar_{2 n}, \hbar_{2 n+1}\right)= & \delta\left(J v_{2 n}, K v_{2 n+1}\right) \leq \eta_{1} \delta\left(f v_{2 n}, f v_{2 n+1}\right) \\
& +\eta_{2} \frac{\delta\left(f v_{2 n}, J v_{2 n}\right) \delta\left(f v_{2 n+1}, K v_{2 n+1}\right)}{1+\delta\left(f v_{2 n}, f v_{2 n+1}\right)} \\
& +\eta_{3} \frac{\left[\delta\left(f v_{2 n}, J v_{2 n}\right) \delta\left(f v_{2 n}, K v_{2 n+1}\right)+\delta\left(f v_{2 n+1}, K v_{2 n+1}\right) \delta\left(f v_{2 n+1}, J v_{2 n}\right)\right]}{\delta\left(f v_{2 n}, K v_{2 n+1}\right)+\delta\left(f v_{2 n+1}, J v_{2 n}\right)} \\
& +\eta_{4}\left[\delta\left(f v_{2 n}, J v_{2 n}\right)+\delta\left(f v_{2 n+1}, K v_{2 n+1}\right)+\delta\left(f v_{2 n}, K v_{2 n+1}\right)\right. \\
& \left.+\delta\left(f v_{2 n+1}, J v_{2 n}\right)\right]=\eta_{1} \delta\left(\hbar_{2 n-1}, \hbar_{2 n}\right)+\eta_{2} \frac{\delta\left(\hbar_{2 n-1}, \hbar_{2 n}\right) \delta\left(\hbar_{2 n}, \hbar_{2 n+1}\right)}{1+\delta\left(\hbar_{2 n-1}, \hbar_{2 n}\right)} \\
& +\eta_{3} \frac{\left[\delta\left(\hbar_{2 n-1}, \hbar_{2 n}\right) \delta\left(\hbar_{2 n-1}, \hbar_{2 n+1}\right)+\delta\left(\hbar_{2 n}, \hbar_{2 n+1}\right) \delta\left(\hbar_{2 n}, \hbar_{2 n}\right)\right]}{\delta\left(\hbar_{2 n-1}, \hbar_{2 n+1}\right)+\delta\left(\hbar_{2 n}, \hbar_{2 n}\right)} \\
& +\eta_{4}\left[\delta\left(\hbar_{2 n-1}, \hbar_{2 n}\right)+\delta\left(\hbar_{2 n}, \hbar_{2 n+1}\right)+\delta\left(\hbar_{2 n-1}, \hbar_{2 n+1}\right)+\delta\left(\hbar_{2 n}, \hbar_{2 n}\right)\right] .
\end{aligned}
$$

This implies that

$$
\begin{aligned}
\left|\delta\left(\hbar_{2 n}, \hbar_{2 n+1}\right)\right| \leq & \eta_{1}\left|\delta\left(\hbar_{2 n-1}, \hbar_{2 n}\right)\right|+\eta_{2} \frac{\left|\delta\left(\hbar_{2 n-1}, \hbar_{2 n}\right)\right|\left|\delta\left(\hbar_{2 n}, \hbar_{2 n+1}\right)\right|}{\left|1+\delta\left(\hbar_{2 n-1}, \hbar_{2 n}\right)\right|} \\
& +\eta_{3} \frac{\left[\left|\delta\left(\hbar_{2 n-1}, \hbar_{2 n}\right)\right|\left|\delta\left(\hbar_{2 n-1}, \hbar_{2 n+1}\right)\right|+\left|\delta\left(\hbar_{2 n}, \hbar_{2 n+1}\right)\right|\left|\delta\left(\hbar_{2 n}, \hbar_{2 n}\right)\right|\right]}{\left|\delta\left(\hbar_{2 n-1}, \hbar_{2 n+1}\right)\right|+\left|\delta\left(\hbar_{2 n}, \hbar_{2 n}\right)\right|} \\
& +\eta_{4}\left[\left|\delta\left(\hbar_{2 n-1}, \hbar_{2 n}\right)\right|+\left|\delta\left(\hbar_{2 n}, \hbar_{2 n+1}\right)\right|+\left|\delta\left(\hbar_{2 n-1}, \hbar_{2 n+1}\right)\right|\right. \\
& \left.+\left|\delta\left(\hbar_{2 n}, \hbar_{2 n}\right)\right|\right] .
\end{aligned}
$$

Now by using triangular inequality of $(V, \delta)$ and after simplification, we get that

$$
\left|\delta\left(\hbar_{2 n}, \hbar_{2 n+1}\right)\right| \leq\left(\frac{\eta_{1}+\eta_{3}+\eta_{4}+\eta_{4} b}{1-\eta_{2}-\eta_{4}-\eta_{4} b}\right)\left|\delta\left(\hbar_{2 n-1}, \hbar_{2 n}\right)\right| .
$$

Again by view of (48) and (49),

$$
\begin{aligned}
\delta\left(\hbar_{2 n-1}, \hbar_{2 n}\right)= & \delta\left(K v_{2 n-1}, J v_{2 n}\right)=\delta\left(J v_{2 n}, K v_{2 n-1}\right) \\
\leq & \eta_{1} \delta\left(f v_{2 n}, f v_{2 n-1}\right)+\eta_{2} \frac{\delta\left(f v_{2 n}, J v_{2 n}\right) \delta\left(f v_{2 n-1}, K v_{2 n-1}\right)}{1+\delta\left(f v_{2 n}, f v_{2 n-1}\right)} \\
& +\eta_{3} \frac{\left[\delta\left(f v_{2 n}, J v_{2 n}\right) \delta\left(f v_{2 n}, K v_{2 n-1}\right)+\delta\left(f v_{2 n-1}, K v_{2 n-1}\right) \delta\left(f v_{2 n-1}, J v_{2 n}\right)\right]}{\delta\left(f v_{2 n}, K v_{2 n-1}\right)+\delta\left(f v_{2 n-1}, J v_{2 n}\right)} \\
& +\eta_{4}\left[\delta\left(f v_{2 n}, J v_{2 n}\right)+\delta\left(f v_{2 n-1}, K v_{2 n-1}\right)+\delta\left(f v_{2 n}, K v_{2 n-1}\right)\right. \\
& \left.+\delta\left(f v_{2 n-1}, J v_{2 n}\right)\right]=\eta_{1} \delta\left(\hbar_{2 n-1}, \hbar_{2 n-2}\right) \\
& +\eta_{2} \frac{\delta\left(\hbar_{2 n-1}, \hbar_{2 n}\right) \delta\left(\hbar_{2 n-2}, \hbar_{2 n-1}\right)}{1+\delta\left(\hbar_{2 n-1}, \hbar_{2 n-2}\right)} \\
& +\eta_{3} \frac{\left[\delta\left(\hbar_{2 n-1}, \hbar_{2 n}\right) \delta\left(\hbar_{2 n-1}, \hbar_{2 n-1}\right)+\delta\left(\hbar_{2 n-2}, \hbar_{2 n-1}\right) \delta\left(\hbar_{2 n-2}, \hbar_{2 n}\right)\right]}{\delta\left(\hbar_{2 n-1}, \hbar_{2 n-1}\right)+\delta\left(\hbar_{2 n-2}, \hbar_{2 n}\right)} \\
& +\eta_{4}\left[\delta\left(\hbar_{2 n-1}, \hbar_{2 n}\right)+\delta\left(\hbar_{2 n-2}, \hbar_{2 n-1}\right)+\delta\left(\hbar_{2 n-1}, \hbar_{2 n-1}\right)+\delta\left(\hbar_{2 n-2}, \hbar_{2 n}\right)\right] .
\end{aligned}
$$

This implies that

$$
\begin{aligned}
\left|\delta\left(\hbar_{2 n-1}, \hbar_{2 n}\right)\right| \leq & \eta_{1}\left|\delta\left(\hbar_{2 n-1}, \hbar_{2 n-2}\right)\right|+\eta_{2} \frac{\left|\delta\left(\hbar_{2 n-1}, \hbar_{2 n}\right)\right|\left|\delta\left(\hbar_{2 n-2}, \hbar_{2 n-1}\right)\right|}{\left|1+\delta\left(\hbar_{2 n-1}, \hbar_{2 n-2}\right)\right|} \\
& +\eta_{3} \frac{\left[\left|\delta\left(\hbar_{2 n-1}, \hbar_{2 n}\right)\right|\left|\delta\left(\hbar_{2 n-1}, \hbar_{2 n-1}\right)\right|+\left|\delta\left(\hbar_{2 n-2}, \hbar_{2 n-1}\right)\right|\left|\delta\left(\hbar_{2 n-2}, \hbar_{2 n}\right)\right|\right]}{\left|\delta\left(\hbar_{2 n-1}, \hbar_{2 n-1}\right)\right|+\left|\delta\left(\hbar_{2 n-2}, \hbar_{2 n}\right)\right|} \\
& +\eta_{4}\left[\left|\delta\left(\hbar_{2 n-1}, \hbar_{2 n}\right)\right|+\left|\delta\left(\hbar_{2 n-2}, \hbar_{2 n-1}\right)\right|+\left|\delta\left(\hbar_{2 n-1}, \hbar_{2 n-1}\right)\right|\right. \\
& \left.+\left|\delta\left(\hbar_{2 n-2}, \hbar_{2 n}\right)\right|\right] .
\end{aligned}
$$

By using triangular inequality of $(V, \delta)$ and after simplification we get that

$$
\left|\delta\left(\hbar_{2 n-1}, \hbar_{2 n}\right)\right| \leq\left(\frac{\eta_{1}+\eta_{3}+\eta_{4}+\eta_{4} b}{1-\eta_{2}-\eta_{4}-\eta_{4} b}\right)\left|\delta\left(\hbar_{2 n-2}, \hbar_{2 n-1}\right)\right| .
$$

Now from (55) and (52) and by induction, we have

$$
\begin{aligned}
\left|\delta\left(\hbar_{2 n}, \hbar_{2 n+1}\right)\right| & \leq q\left|\delta\left(\hbar_{2 n-1}, \hbar_{2 n}\right)\right| \leq q^{2}\left|\delta\left(\hbar_{2 n-2}, \hbar_{2 n-1}\right)\right| \\
& \leq \cdots \leq q^{2 n}\left|\delta\left(\hbar_{0}, \hbar_{1}\right)\right|
\end{aligned}
$$

where $q=\left(\eta_{1}+\eta_{3}+\eta_{4}+\eta_{4} b\right) /\left(1-\eta_{2}-\eta_{4}-\eta_{4} b\right)<1$. Next, we have to show that $\left\{\hbar_{n}\right\}$ is a Cauchy sequence. Let $m, n$ $\in \mathbb{N}$ and $m>n$. Then, we have

$$
\begin{aligned}
\left|\delta\left(\hbar_{n}, \hbar_{m}\right)\right| & \leq b\left|\delta\left(\hbar_{n}, \hbar_{n+1}\right)\right|+b\left|\delta\left(\hbar_{n+1}, \hbar_{m}\right)\right| \\
& \leq b\left|\delta\left(\hbar_{n}, \hbar_{n+1}\right)\right|+b^{2}\left|\delta\left(\hbar_{n+1}, \hbar_{n+2}\right)\right|+\cdots+b^{m-n}\left|\delta\left(\hbar_{m-1}, \hbar_{m}\right)\right| \\
& \leq b q^{n}\left|\delta\left(\hbar_{0}, \hbar_{1}\right)\right|+b^{2} q^{n+1}\left|\delta\left(\hbar_{0}, \hbar_{1}\right)\right|+\cdots+b^{m-n} q^{m-1}\left|\delta\left(\hbar_{0}, \hbar_{1}\right)\right| \\
& \leq\left[b q^{n}+b^{2} q^{n+1}+\cdots+b^{m-n} q^{m-1}\right]\left|\delta\left(\hbar_{0}, \hbar_{1}\right)\right| \\
& =b q^{n}\left[1+b q+b^{2} q^{2} \cdots+b^{m-(n+1)} q^{m-(n+1)}\right]\left|\delta\left(\hbar_{0}, \hbar_{1}\right)\right| \\
& =b q^{n} \sum_{t=0}^{m-(n+1)} b^{t} q^{t}\left|\delta\left(\hbar_{0}, \hbar_{1}\right)\right| \leq b q^{n} \sum_{t=0}^{\infty} b^{t} q^{t}\left|\delta\left(\hbar_{0}, \hbar_{1}\right)\right| \\
& =\frac{b q^{n}}{1-b q}\left|\delta\left(\hbar_{0}, \hbar_{1}\right)\right| \longrightarrow 0, \text { as } n \longrightarrow \infty .
\end{aligned}
$$


Hence, $\left\{\hbar_{n}\right\}$ is a Cauchy sequence. Since $V$ is complete, there exists some $p \in V$, such that $\hbar_{n} \longrightarrow p$, as $n \longrightarrow \infty$, and from (49), we have that

$$
\lim _{n \longrightarrow \infty} f v_{2 n+1}=p, \lim _{n \longrightarrow \infty} J v_{2 n}=p, \lim _{n \longrightarrow \infty} K v_{2 n+1}=p .
$$

$f$ is a continuous self-mapping on $V$, so that

$\lim _{n \longrightarrow \infty} f\left(f v_{2 n+1}\right)=f p, \lim _{n \longrightarrow \infty} f\left(J v_{2 n}\right)=f p, \lim _{n \longrightarrow \infty} f\left(K v_{2 n+1}\right)=f p$.

Since $(f, J)$ is compatible and for some sequence $\left\{v_{2 n}\right\}$ in $V$, we have that

$$
\lim _{n \rightarrow \infty}\left|\delta\left(J\left(f v_{2 n}\right), f\left(J v_{2 n}\right)\right)\right|=0 .
$$

From (59), (60), and by using Lemma 7, we get that

$$
\lim _{n \longrightarrow \infty} J\left(f v_{2 n}\right)=f p .
$$

Now, we have to show that $f p=p$. So, by putting $v_{1}=f$ $v_{2 n}$ and $v_{2}=v_{2 n+1}$ in (48),

$$
\begin{aligned}
& \delta\left(J\left(f v_{2 n}\right), K v_{2 n+1}\right) \leq \eta_{1} \delta\left(f\left(f v_{2 n}\right), f v_{2 n+1}\right)+\eta_{2} \frac{\delta\left(f\left(f v_{2 n}\right), J\left(f v_{2 n}\right)\right) \delta\left(f v_{2 n+1}, K v_{2 n+1}\right)}{1+\delta\left(f\left(f v_{2 n}\right), f v_{2 n+1}\right)} \\
& \quad+\eta_{3} \frac{\left[\delta\left(f\left(f v_{2 n}\right), J\left(f v_{2 n}\right)\right) \delta\left(f\left(f v_{2 n}\right), K v_{2 n+1}\right)+\delta\left(f v_{2 n+1}, K v_{2 n+1}\right) \delta\left(f v_{2 n+1}, J\left(f v_{2 n}\right)\right)\right]}{\delta\left(f\left(f v_{2 n}\right), K v_{2 n+1}\right)+\delta\left(f v_{2 n+1}, J\left(f v_{2 n}\right)\right)} \\
& \quad+\eta_{4}\left[\delta\left(f\left(f v_{2 n}\right), J\left(f v_{2 n}\right)\right)+\delta\left(f v_{2 n+1}, K v_{2 n+1}\right)+\delta\left(f\left(f v_{2 n}\right), K v_{2 n+1}\right)\right) \\
& \left.\quad+\delta\left(f v_{2 n+1}, J\left(f v_{2 n}\right)\right)\right] .
\end{aligned}
$$

This implies that

$$
\begin{aligned}
& \left|\delta\left(J\left(f v_{2 n}\right), K v_{2 n+1}\right)\right| \leq \eta_{1}\left|\delta\left(f\left(f v_{2 n}\right), f v_{2 n+1}\right)\right|+\eta_{2} \frac{\left|\delta\left(f\left(f v_{2 n}\right), J\left(f v_{2 n}\right)\right)\right|\left|\delta\left(f v_{2 n+1}, K v_{2 n+1}\right)\right|}{\left|1+\delta\left(f\left(f v_{2 n}\right), f v_{2 n+1}\right)\right|} \\
& \quad+\eta_{3} \frac{\left[\left|\delta\left(f\left(f v_{2 n}\right), J\left(f v_{2 n}\right)\right)\right|\left|\delta\left(f\left(f v_{2 n}\right), K v_{2 n+1}\right)\right|+\left|\delta\left(f v_{2 n+1}, K v_{2 n+1}\right)\right|\left|\delta\left(f v_{2 n+1}, J\left(f v_{2 n}\right)\right)\right|\right]}{\left|\delta\left(f\left(f v_{2 n}\right), K v_{2 n+1}\right)\right|+\left|\delta\left(f v_{2 n+1}, J\left(f v_{2 n}\right)\right)\right|} \\
& \quad+\eta_{4}\left[\left|\delta\left(f\left(f v_{2 n}\right), J\left(f v_{2 n}\right)\right)\right|+\left|\delta\left(f v_{2 n+1}, K v_{2 n+1}\right)\right|+\left|\delta\left(f\left(f v_{2 n}\right), K v_{2 n+1}\right)\right|\right. \\
& \left.\quad+\mid \delta\left(f v_{2 n+1}, J\left(f v_{2 n}\right)\right)\right] .
\end{aligned}
$$

Applying $\lim _{n \longrightarrow \infty}$ on both sides and using (58), (59), and (61), we get that

$$
\begin{aligned}
|\delta(f p, p)| \leq & \eta_{1}|\delta(f p, p)|+\eta_{2} \frac{|\delta(f p, f p)||\delta(p, p)|}{|1+\delta(f p, p)|} \\
& +\eta_{3} \frac{[|\delta(f p, f p)||\delta(f p, p)|+|\delta(p, p)||\delta(p, f p)|]}{|\delta(f p, p)|+|\delta(p, f p)|} \\
& +\eta_{4}[|\delta(f p, f p)|+|\delta(p, p)|+|\delta(f p, p)|+|\delta(p, f p)|] \\
= & \left(\eta_{1}+2 \eta_{4}\right)|\delta(f p, p)| .
\end{aligned}
$$

This implies that $\left(1-\eta_{1}-2 \eta_{4}\right)|\delta(f p, p)| \leq 0$. Since $(1-$ $\left.\eta_{1}-2 \eta_{4}\right) \neq 0 \Rightarrow|\delta(f p, p)|=0$, hence,

$$
f p=p .
$$

Next, we have to show that $J p=p$, and by using (48),

$$
\begin{aligned}
\delta\left(J p, f v_{2 n+2}\right)= & \delta\left(J p, K v_{2 n+1}\right) \leq \eta_{1} \delta\left(f p, f v_{2 n+1}\right) \\
& +\eta_{2} \frac{\delta(f p, J p) \delta\left(f v_{2 n+1}, K v_{2 n+1}\right)}{1+\delta\left(f p, f v_{2 n+1}\right)} \\
& +\eta_{3} \frac{\left[\delta(f p, J p) \delta\left(f p, K v_{2 n+1}\right)+\delta\left(f v_{2 n+1}, K v_{2 n+1}\right) \delta\left(f v_{2 n+1}, J p\right)\right]}{\delta\left(f p, K v_{2 n+1}\right)+\delta\left(f v_{2 n+1}, J p\right)} \\
& +\eta_{4}\left[\delta(f p, J p)+\delta\left(f v_{2 n+1}, K v_{2 n+1}\right)+\delta\left(f p, K v_{2 n+1}\right)\right. \\
& \left.+\delta\left(f v_{2 n+1}, J p\right)\right] .
\end{aligned}
$$

This implies that

$$
\begin{aligned}
\left|\delta\left(J p, f v_{2 n+2}\right)\right| \leq & \eta_{1}\left|\delta\left(f p, f v_{2 n+1}\right)\right|+\eta_{2} \frac{|\delta(f p, J p)|\left|\delta\left(f v_{2 n+1}, K v_{2 n+1}\right)\right|}{\left|1+\delta\left(f p, f v_{2 n+1}\right)\right|} \\
& +\eta_{3} \frac{\left[|\delta(f p, J p)|\left|\delta\left(f p, K v_{2 n+1}\right)\right|+\left|\delta\left(f v_{2 n+1}, K v_{2 n+1}\right)\right|\left|\delta\left(f v_{2 n+1}, J p\right)\right|\right]}{\left|\delta\left(f p, K v_{2 n+1}\right)\right|+\left|\delta\left(f v_{2 n+1}, J p\right)\right|} \\
& +\eta_{4}\left[|\delta(f p, J p)|+\left|\delta\left(f v_{2 n+1}, K v_{2 n+1}\right)\right|+\left|\delta\left(f p, K v_{2 n+1}\right)\right|\right. \\
& \left.+\left|\delta\left(f v_{2 n+1}, J p\right)\right|\right] .
\end{aligned}
$$

Applying $\lim _{n \rightarrow \infty}$ on both sides and using (58) and (65), we have

$$
\begin{aligned}
|\delta(J p, p)| \leq & \eta_{1}|\delta(f p, p)|+\eta_{2} \frac{|\delta(f p, J p)||\delta(p, p)|}{|1+\delta(f p, p)|} \\
& +\eta_{3} \frac{[|\delta(f p, J p)||\delta(f p, p)|+|\delta(p, p)||\delta(p, J p)|]}{|\delta(f p, p)|+|\delta(p, J p)|} \\
& +\eta_{4}[|\delta(f p, J p)|+|\delta(p, p)|+|\delta(f p, p)|+|\delta(p, J p)|] \\
= & \eta_{1}|\delta(p, p)|+\eta_{2} \frac{|\delta(p, J p)||\delta(p, p)|}{|1+\delta(p, p)|} \\
& +\eta_{3} \frac{[|\delta(p, J p)||\delta(p, p)|+|\delta(p, p)||\delta(p, J p)|]}{|\delta(p, p)|+|\delta(p, J p)|} \\
& +\eta_{4}[|\delta(p, J p)|+|\delta(p, p)|+|\delta(p, p)|+|\delta(p, J p)|] .
\end{aligned}
$$

Thus, we get that $|\delta(J p, p)| \leq 2 \eta_{4}|\delta(p, J p)| \Rightarrow\left(1-2 \eta_{4}\right) \mid \delta$ $(J p, p) \mid \leq 0$. Since $\left(1-2 \eta_{4}\right) \neq 0$, as $\left(\eta_{1}+\eta_{2}+\eta_{3}+2 \eta_{4}+2 \eta_{4} b\right)$ $<1$, therefore $|\delta(J p, p)|=0$. Hence,

$$
J p=p .
$$

Now, we have to show that $K p=p$, and by using (48),

$$
\begin{aligned}
\delta\left(f v_{2 n+1}, K p\right)= & \delta\left(J v_{2 n}, K p\right) \leq \eta_{1} \delta\left(f v_{2 n}, f p\right) \\
& +\eta_{2} \frac{\delta\left(f v_{2 n}, J v_{2 n}\right) \delta(f p, K p)}{1+\delta\left(f v_{2 n}, f p\right)} \\
& +\eta_{3} \frac{\left[\delta\left(f v_{2 n}, J v_{2 n}\right) \delta\left(f v_{2 n}, K p\right)+\delta(f p, K p) \delta\left(f p, J v_{2 n}\right)\right]}{\delta\left(f v_{2 n}, K p\right)+\delta\left(f p, J v_{2 n}\right)} \\
& +\eta_{4}\left[\delta\left(f v_{2 n}, J v_{2 n}\right)+\delta(f p, K p)+\delta\left(f v_{2 n}, K p\right)\right. \\
& \left.+\delta\left(f p, J v_{2 n}\right)\right] .
\end{aligned}
$$


This implies that

$$
\begin{aligned}
\left|\delta\left(f v_{2 n+1}, K p\right)\right| \leq & \eta_{1}\left|\delta\left(f v_{2 n}, f p\right)\right|+\eta_{2} \frac{\left|\delta\left(f v_{2 n}, J v_{2 n}\right)\right||\delta(f p, K p)|}{\left|1+\delta\left(f v_{2 n}, f p\right)\right|} \\
& +\eta_{3} \frac{\left[\delta\left(f v_{2 n}, J v_{2 n}\right)|| \delta\left(f v_{2 n}, K p\right)|+| \delta(f p, K p)|| \delta\left(f p, J v_{2 n}\right) \mid\right]}{\left|\delta\left(f v_{2 n}, K p\right)\right|+\left|\delta\left(f p, J v_{2 n}\right)\right|} \\
& +\eta_{4}\left[\left|\delta\left(f v_{2 n}, J v_{2 n}\right)\right|+|\delta(f p, K p)|+\left|\delta\left(f v_{2 n}, K p\right)\right|\right. \\
& \left.+\left|\delta\left(f p, J v_{2 n}\right)\right|\right] .
\end{aligned}
$$

Applying $\lim _{n \longrightarrow \infty}$ on both sides and by view of (58) and (65), we have that

$$
\begin{aligned}
|\delta(p, K p)| \leq & \eta_{1}|\delta(p, f p)|+\eta_{2} \frac{|\delta(p, p)||\delta(f p, K p)|}{|1+\delta(p, f p)|} \\
& +\eta_{3} \frac{[|\delta(p, p)||\delta(p, K p)|+|\delta(f p, K p)||\delta(f p, p)|]}{|\delta(p, K p)|+|\delta(f p, p)|} \\
& +\eta_{4}[|\delta(p, p)|+|\delta(f p, K p)|+|\delta(p, K p)|+|\delta(f p, p)|] \\
= & 2 \eta_{4}|\delta(p, K p)| .
\end{aligned}
$$

This implies that $\left(1-2 \eta_{4}\right)|\delta(p, K p)| \leq 0$. Since $\left(1-2 \eta_{4}\right)$ $\neq 0$, therefore $|\delta(p, K p)|=0$. Hence,

$$
K p=p
$$

Thus, from (65), (69), and (73), we get that $p$ is a common fixed point of $f, J$ and $K$, i.e.,

$$
f p=J p=K p=p
$$

Uniqueness: we contrary suppose that $p^{*}$ is another common fixed point of $f, J$ and $K$ such that $f p^{*}=J p^{*}=K p^{*}=p^{*}$. Now, by (48),

$$
\begin{aligned}
\delta\left(p, p^{*}\right)= & \delta\left(J p, K p^{*}\right) \leq \eta_{1} \delta\left(f p, f p^{*}\right)+\eta_{2} \frac{\delta(f p, J p) \delta\left(f p^{*}, K p^{*}\right)}{1+\delta\left(f p, f p^{*}\right)} \\
& +\eta_{3} \frac{\left[\delta(f p, J p) \delta\left(f p, K p^{*}\right)+\delta\left(f p^{*}, K p^{*}\right) \delta\left(f p^{*}, J p\right)\right]}{\delta\left(f p, K p^{*}\right)+\delta\left(f p^{*}, J p\right)} \\
& +\eta_{4}\left[\delta(f p, J p)+\delta\left(f p^{*}, K p^{*}\right)+\delta\left(f p, K p^{*}\right)+\delta\left(f p^{*}, J p\right)\right] \\
= & \left(\eta_{1}+2 \eta_{4}\right) \delta\left(p, p^{*}\right) .
\end{aligned}
$$

This implies that $\left|\delta\left(p, p^{*}\right)\right| \leq\left(\eta_{1}+2 \eta_{4}\right)\left|\delta\left(p, p^{*}\right)\right| \Rightarrow(1-$ $\left.\eta_{1}-2 \eta_{4}\right)\left|\delta\left(p, p^{*}\right)\right| \leq 0$. Since $\left(1-\eta_{1}-\eta_{4}\right) \neq 0$, therefore $\mid \delta(p$, $\left.p^{*}\right) \mid \leq 0 \longrightarrow p=p^{*}$. Hence, we proved that $f, J$ and $K$ have a unique common fixed point in $V$.

Example 3. Let $V=[0, \infty)$ and $\delta: V \times V \longrightarrow \mathbb{C}$ defined by $\delta$ $\left(v_{1}, v_{2}\right)=3\left|v_{1}-v_{2}\right|^{2} / 13+i\left(3\left|v_{1}-v_{2}\right|^{2} / 13\right)$ for all $v_{1}, v_{2} \in V$ is a b-metric on $V$ and $(V, \delta)$ is a complex-valued b-metric space. Now, first, we show that $V$ is a $b$-metric with $b=2$, so that

$$
\begin{aligned}
\delta\left(v_{1}, v_{2}\right)= & \frac{3\left|v_{1}-v_{2}\right|^{2}}{13}+i \frac{3\left|v_{1}-v_{2}\right|^{2}}{13} \leq \frac{3\left|\left(v_{1}-v_{3}\right)+\left(v_{3}-v_{2}\right)\right|^{2}}{13} \\
& +i \frac{3\left|\left(v_{1}-v_{3}\right)+\left(v_{3}-v_{2}\right)\right|^{2}}{13} \\
\leq & {\left[\frac{3\left|v_{1}-v_{3}\right|^{2}}{13}+\frac{3\left|v_{3}-v_{2}\right|^{2}}{13}+\frac{3}{13}\left(2\left|v_{1}-v_{3}\right|\left|v_{3}-v_{2}\right|\right)\right] } \\
& +i\left[\frac{3\left|v_{1}-v_{3}\right|^{2}}{13}+\frac{3\left|v_{3}-v_{2}\right|^{2}}{13}+\frac{3}{13}\left(2\left|v_{1}-v_{3}\right|\left|v_{3}-v_{2}\right|\right)\right] \\
\leq & {\left[\frac{3\left|v_{1}-v_{3}\right|^{2}}{13}+\frac{3\left|v_{3}-v_{2}\right|^{2}}{13}+\frac{3\left|v_{1}-v_{3}\right|^{2}}{13}+\frac{3\left|v_{3}-v_{2}\right|^{2}}{13}\right] } \\
& +i\left[\frac{3\left|v_{1}-v_{3}\right|^{2}}{13}+\frac{3\left|v_{3}-v_{2}\right|^{2}}{13}+\frac{3\left|v_{1}-v_{3}\right|^{2}}{13}+\frac{3\left|v_{3}-v_{2}\right|^{2}}{13}\right] \\
= & 2\left[\frac{3\left|v_{1}-v_{3}\right|^{2}}{13}+\frac{3\left|v_{3}-v_{2}\right|^{2}}{13}\right]^{2}+i 2\left[\frac{3\left|v_{1}-v_{3}\right|^{2}}{13}+\frac{3\left|v_{3}-v_{2}\right|^{2}}{13}\right] \\
= & 2\left[\frac{3\left|v_{1}-v_{3}\right|^{2}}{13}+i \frac{3\left|v_{1}-v_{3}\right|^{2}}{13}+\frac{3\left|v_{3}-v_{2}\right|^{2}}{13}+i \frac{3\left|v_{3}-v_{2}\right|^{2}}{13}\right]^{2} \\
= & 2\left[\delta\left(v_{1}, v_{3}\right)+\delta\left(v_{3}, v_{2}\right)\right] .
\end{aligned}
$$

That is, $\delta\left(v_{1}, v_{2}\right) \leq b\left[\delta\left(v_{1}, v_{3}\right)+\delta\left(v_{3}, v_{2}\right)\right]$, with $b=2$.

Define $J, K, f: V \longrightarrow V$ by $J v_{1}=K v_{1}=\ln \left(1+v_{1} / 3+v_{1}\right)$ , and $f v_{1}=e^{3 v_{1}}-1$.

Notice that

$$
\left\{\begin{array}{c}
\left|\delta\left(f v_{1}, f v_{2}\right)\right|, \frac{\left|\delta\left(f v_{1}, J v_{1}\right)\right|\left|\delta\left(f v_{2}, K v_{2}\right)\right|}{\left|1+\delta\left(f v_{1}, f v_{2}\right)\right|}, \frac{\left[\left|\delta\left(f v_{1}, J v_{1}\right)\right|\left|\delta\left(f v_{1}, K v_{2}\right)\right|+\left|\delta\left(f v_{2}, K v_{2}\right)\right|\left|\delta\left(f v_{2}, J v_{1}\right)\right|\right]}{\left|\delta\left(f v_{1}, K v_{2}\right)\right|+\left|\delta\left(f v_{2}, J v_{1}\right)\right|} \\
{\left[\left|\delta\left(f v_{1}, J v_{1}\right)\right|+\left|\delta\left(f v_{2}, K v_{2}\right)\right|+\left|\delta\left(f v_{1}, K v_{2}\right)\right|+\left|\delta\left(f v_{2}, J v_{1}\right)\right|\right]}
\end{array}\right\} \geq 0
$$


in all regards. It is enough to show that $\delta\left(J v_{1}, K v_{2}\right) \leq \eta_{1} \delta(f$ $\left.v_{1}, f v_{2}\right)$, for all $v_{1}, v_{2} \in[0, \infty)$ and $\eta_{1}, \eta_{2}, \eta_{3} \in[0,1), \eta_{4} \in[0,1$ 14) such that $\left(\eta_{1}+\eta_{2}+\eta_{3}+2 \eta_{4}+2 \eta_{4} b\right)<1$, where $b \geq 1$, and we have

$$
\begin{aligned}
\delta\left(J v_{1}, K v_{2}\right)= & {\left[\frac{3\left|J v_{1}-K v_{2}\right|^{2}}{13}+i \frac{3\left|J v_{1}-K v_{2}\right|^{2}}{13}\right] } \\
= & {\left[\frac{3\left|\ln \left(1+v_{1} / 3+v_{1}\right)-\ln \left(1+v_{2} / 3+v_{2}\right)\right|^{2}}{13}\right.} \\
& \left.+i \frac{3\left|\ln \left(1+v_{1} / 3+v_{1}\right)-\ln \left(1+v_{2} / 3+v_{2}\right)\right|^{2}}{13}\right] \\
\leq & {\left[\frac{3\left|v_{1} / 3+v_{1}-v_{2} / 3+v_{2}\right|^{2}}{13}+i \frac{3\left|v_{1} / 3+v_{1}-v_{2} / 3+v_{2}\right|^{2}}{13}\right] } \\
\leq & {\left[\frac{3\left|3 v_{1}-3 v_{2} / 9\right|^{2}}{13}+i \frac{3\left|3 v_{1}-3 v_{2} / 9\right|^{2}}{13}\right] } \\
= & \frac{1}{9^{2}}\left[\frac{3\left|3 v_{1}-3 v_{2}\right|^{2}}{13}+i \frac{3\left|3 v_{1}-3 v_{2}\right|^{2}}{13}\right] \\
\leq & \frac{1}{81}\left[\frac{3\left|e^{3 v_{1}}-e^{3 v_{2}}\right|^{2}}{13}+i \frac{3\left|e^{3 v_{1}}-e^{3 v_{2}}\right|^{2}}{13}\right] .
\end{aligned}
$$

And

$$
\begin{aligned}
\delta\left(f v_{1}, f v_{2}\right) & =\left[\frac{3\left|f v_{1}-f v_{2}\right|^{2}}{13}+i \frac{3\left|f v_{1}-f v_{2}\right|^{2}}{13}\right] \\
& =\left[\frac{3\left|\left(e^{3 v_{1}}-1\right)-\left(e^{3 v_{2}}-1\right)\right|^{2}}{13}+i \frac{3\left|\left(e^{3 v_{1}}-1\right)-\left(e^{3 v_{2}}-1\right)\right|^{2}}{13}\right] \\
& =\left[\frac{3\left|e^{3 v_{1}}-e^{3 v_{2}}\right|^{2}}{13}+i \frac{3\left|e^{3 v_{1}}-e^{3 v_{2}}\right|^{2}}{13}\right] .
\end{aligned}
$$

For $v_{1}, v_{2} \in[0, \infty)$, we discuss different cases with $\eta_{1}=1$ $15, \eta_{2}=1 / 4, \eta_{3}=1 / 10, \eta_{4}=1 / 20$, and $b=2$. Notice that $\eta_{1}+$ $\eta_{2}+\eta_{3}+2 \eta_{4} b=1 / 5+1 / 4+1 / 10+2(1 / 20) 2<1$.

Case 1. Let $v_{1}=0, v_{2}=0$. Then, from (78) and (79), directly we get that $\delta\left(J v_{1}, K v_{2}\right) \leq \eta_{1} \delta\left(f v_{1}, f v_{2}\right)$. Hence, (48) is satisfied with $\eta_{1}=1 / 5, \eta_{2}=1 / 4, \eta_{3}=1 / 10, \eta_{4}=1 / 20$, and $b=2$.

Case 2. Let $v_{1}=0, v_{2}=1$; then, from (78) and (79), we find $\delta\left(J v_{1}, K v_{2}\right) \leq \eta_{1} \delta\left(f \mathrm{v}_{1}, f v_{2}\right)$ is satisfied with $\eta_{1}=1 / 5$, as

$$
\frac{1}{81}\left[\frac{3\left|1-e^{3}\right|^{2}}{13}+i \frac{3\left|1-e^{3}\right|^{2}}{13}\right] \leq \eta_{1}\left[\frac{3\left|1-e^{3}\right|^{2}}{13}+i \frac{3\left|1-e^{3}\right|^{2}}{13}\right] .
$$

By using $\eta_{1}=1 / 5$ and after simplifying, we get that

$$
\begin{aligned}
\frac{1}{81} & {\left[\frac{3|-19.0855|^{2}}{13}+i \frac{3|-19.0855|^{2}}{13}\right] } \\
& \leq \frac{1}{5}\left[\frac{3|-19.0855|^{2}}{13}+i \frac{3|-19.0855|^{2}}{13}\right] 1.04[1+i] \\
& \leq 16.81[1+i] .
\end{aligned}
$$

Hence, (48) is satisfied with $\eta_{1}=1 / 5, \eta_{2}=1 / 4, \eta_{3}=1 / 10$, $\eta_{4}=1 / 20$, and $b=2$.

Case 3. Let $v_{1}=1 / 2, v_{2}=1 / 4$; then, from (78) and (79), we find $\delta\left(J v_{1}, K v_{2}\right) \leq \eta_{1} \delta\left(f v_{1}, f v_{2}\right)$, is true for $\eta_{1}=1 / 5$, as

$$
\begin{aligned}
\frac{1}{81} & {\left[\frac{3\left|e^{3 / 2}-e^{3 / 4}\right|^{2}}{13}+i \frac{3\left|e^{3 / 2}-e^{3 / 4}\right|^{2}}{13}\right] } \\
& \leq \eta_{1}\left[\frac{3\left|e^{3 / 2}-e^{3 / 4}\right|^{2}}{13}+i \frac{3\left|e^{3 / 2}-e^{3 / 4}\right|^{2}}{13}\right] .
\end{aligned}
$$

By using $\eta_{1}=1 / 5$ and after simplifying, we get that

$$
\begin{aligned}
\frac{1}{81} & {\left[\frac{3|2.3646|^{2}}{13}+i \frac{3|2.3646|^{2}}{13}\right] } \\
& \leq \frac{1}{5}\left[\frac{3|2.3646|^{2}}{13}+i \frac{3|2.3646|^{2}}{13}\right] 0.02[1+i] \leq 0.26[1+i] .
\end{aligned}
$$

Hence, (48) is satisfied with $\eta_{1}=1 / 5, \eta_{2}=1 / 4, \eta_{3}=1 / 10$, $\eta_{4}=1 / 20$, and $b=2$.

Case 4. Let $v_{1}=1 / 2, v_{2}=1$; then, from (78) and (79), we get that $\delta\left(J v_{1}, K v_{2}\right) \leq \eta_{1} \delta\left(f v_{1}, f v_{2}\right)$ is true for $\eta_{1}=1 / 5$, as

$$
\begin{aligned}
\frac{1}{81} & {\left[\frac{3\left|e^{3 / 2}-e^{3}\right|^{2}}{13}+i \frac{3\left|e^{3 / 2}-e^{3}\right|^{2}}{13}\right] } \\
& \leq \eta_{1}\left[\frac{3\left|e^{3 / 2}-e^{3}\right|^{2}}{13}+i \frac{3\left|e^{3 / 2}-e^{3}\right|^{2}}{13}\right] .
\end{aligned}
$$

By using $\eta_{1}=1 / 5$ and after simplifying, we get that

$$
\begin{aligned}
& \frac{1}{81} {\left[\frac{3|-15.6038|^{2}}{13}+i \frac{3|-15.6038|^{2}}{13}\right] } \\
& \quad \leq \frac{1}{5}\left[\frac{3|-15.6038|^{2}}{13}+i \frac{3|-15.6038|^{2}}{13}\right] 0.69[1+i] \\
& \quad \leq 11.24[1+i] .
\end{aligned}
$$

Hence, (48) is satisfied with $\eta_{1}=1 / 5, \eta_{2}=1 / 4, \eta_{3}=1 / 10$, $\eta_{4}=1 / 20$, and $b=2$. 
Case 5. Let $v_{1}=1, v_{2}=5$; then, from (78) and (79), we find $\delta\left(J v_{1}, K v_{2}\right) \leq \eta_{1} \delta\left(f v_{1}, f v_{2}\right)$ is true for $\eta_{1}=1 / 5$, as

$$
\frac{1}{81}\left[\frac{3\left|e^{3}-e^{15}\right|^{2}}{13}+i \frac{3\left|e^{3}-e^{15}\right|^{2}}{13}\right] \leq \eta_{1}\left[\frac{3\left|e^{3}-e^{15}\right|^{2}}{13}+i \frac{3\left|e^{3}-e^{15}\right|^{2}}{13}\right] \text {. }
$$

By using $\eta_{1}=1 / 5$ and after simplifying, we get that

$$
\begin{aligned}
& \frac{1}{81}\left[\frac{3|-3268997.28|^{2}}{13}+i \frac{3|-3268997.28|^{2}}{13}\right] \\
& \quad \leq \frac{1}{5}\left[\frac{3|-3268997.28|^{2}}{13}+i \frac{3|-3268997.28|^{2}}{13}\right] 0.304 \times 10^{11}[1+i] \\
& \quad \leq 4.932 \times 10^{11}[1+i] .
\end{aligned}
$$

Hence, (48) is satisfied with $\eta_{1}=1 / 5, \eta_{2}=1 / 4, \eta_{3}=1 / 10$, $\eta_{4}=1 / 20$, and $b=2$.

Thus, all conditions of Theorem 13 are satisfied with noticing that the point $0 \in V$ remains fixed under mappings $f$, $J$ and $K$ and is indeed unique.

Corollary 14. Let $(V, \delta)$ be a complete complex valued b-metric space and let $J, K, f: V \longrightarrow V$ be three self-mappings satisfying the following:

$$
\begin{aligned}
\delta\left(J v_{1}, K v_{2}\right) \leq & \eta_{1} \delta\left(f v_{1}, f v_{2}\right)+\eta_{2} \\
& \cdot \frac{\left[\delta\left(f v_{1}, J v_{1}\right) \delta\left(f v_{1}, K v_{2}\right)+\delta\left(f v_{2}, K v_{2}\right) \delta\left(f v_{2}, J v_{1}\right)\right]}{1+\delta\left(f v_{1}, f v_{2}\right)} \\
& +\eta_{3}\left[\delta\left(f v_{1}, J v_{1}\right)+\delta\left(f v_{2}, K v_{2}\right)+\delta\left(f v_{1}, K v_{2}\right)\right. \\
& \left.+\delta\left(f v_{2}, J v_{1}\right)\right],
\end{aligned}
$$

for all $v_{1}, v_{2} \in V$ and $\eta_{1}, \eta_{2} \in[0,1), \eta_{3} \in[0,1 / 4)$, such that $\left(\eta_{1}+2 \eta_{2} b+2 \eta_{3}+2 \eta_{3} b\right)<1$, where $b \geq 1$. If $f$ is continuous and $(f, J),(f, K)$ are compatible, then $f, J$ and $K$ have a unique common fixed point in $V$.

\section{Applications}

In this section, we present an integral type application to support our main work. For this purpose, we use the two UITEs to get the existing result of a common solution to verify the validity of our work. Let $V=C\left(\left[k_{1}, k_{2}\right], \mathbb{R}^{n}\right)$ be the set of all real-valued continuous functions defined on $\left[k_{1}, k_{2}\right]$. In the following, we apply Theorem 9 to get the existing result of a common solution by using the two UITEs. Now we are in the position to present a theorem based on the two UITEs to get the existing result of a common solution to support our work.

Theorem 15 (see [23]). Let $V=C\left(\left[k_{1}, k_{2}\right], \mathbb{R}^{n}\right)$, where $\left[k_{1}, k_{2}\right]$ $\subseteq \mathbb{R}$ and $\delta: V \times V \longrightarrow \mathbb{C}$ is defined as

$$
\delta\left(v_{1}, v_{2}\right)=\left\|v_{1}(p)-v_{2}(p)\right\|^{2} \sqrt{1+k_{1}^{2}} e^{i \cot k_{1}},
$$

for all $v_{1}, v_{2} \in V$ and $p \in\left[k_{1}, k_{2}\right]$. Consider the UITEs are

$$
\begin{aligned}
& v_{1}(p)=\int_{k_{1}}^{k_{2}} Q_{1}\left(p, r, v_{1}(r)\right) d r+\hbar_{1}(p), \\
& v_{2}(p)=\int_{k_{1}}^{k_{2}} Q_{2}\left(p, r, v_{2}(r)\right) d r+\hbar_{2}(p),
\end{aligned}
$$

where $r \in\left[k_{1}, k_{2}\right]$. Let $Q_{1}, Q_{2}:\left[k_{1}, k_{2}\right] \times\left[k_{1}, k_{2}\right] \times \mathbb{R}^{n} \longrightarrow \mathbb{R}^{n}$ are such that $D_{v_{1}}, E_{v_{2}} \in V$ for every $v_{1}, v_{2} \in V$, and we have that

$$
\begin{aligned}
& D_{v_{1}}(p)=\int_{k_{1}}^{k_{2}} Q_{1}\left(p, r, v_{1}(r)\right) d r \\
& E_{v_{2}}(p)=\int_{k_{1}}^{k_{2}} Q_{2}\left(p, r, v_{2}(r)\right) d r .
\end{aligned}
$$

If there exists $\mu \in(0,1)$ such that for all $v_{1}, v_{2} \in V$,

$$
\left\|D_{v_{1}}(p)-E_{v_{2}}(p)+\hbar_{1}(p)-\hbar_{2}(p)\right\|^{2} \sqrt{1+k_{1}^{2}} e^{i \cot k_{1}} \leq \mu M\left(v_{1}, v_{2}\right),
$$

where

$M\left(v_{1}, v_{2}\right)=\max \left\{A_{1}\left(v_{1}, v_{2}\right)(p), A_{2}\left(v_{1}, v_{2}\right)(p), A_{3}\left(v_{1}, v_{2}\right)(p), A_{4}\left(v_{1}, v_{2}\right)(p)\right\}$

with

$$
\begin{gathered}
A_{1}\left(v_{1}, v_{2}\right)(p)=\left\|v_{1}(p)-v_{2}(p)\right\|^{2} \sqrt{1+k_{1}^{2}} e^{i} \cot k_{1}, \\
A_{2}\left(v_{1}, v_{2}\right)(p)=\frac{\left\|D_{v_{1}}(p)+\hbar_{1}(p)-v_{1}(p)\right\|^{2}\left\|E_{v_{2}}(p)+\hbar_{2}(p)-v_{2}(p)\right\|^{2}\left(\sqrt{1+k_{1}^{2}} e^{i \cot k_{1}}\right)^{2}}{1+\left\|v_{1}(p)-v_{2}(p)\right\|^{2} \sqrt{1+k_{1}^{2}} e^{i \cot k_{1}}} \\
A_{3}\left(v_{1}, v_{2}\right)(p)=\frac{\left(\left\|D_{v_{1}}(p)+\hbar_{1}(p)-v_{1}(p)\right\|^{2}\left\|E_{v_{2}}(p)+\hbar_{2}(p)-v_{1}(p)\right\|^{2}+\left\|E_{v_{2}}(p)+\hbar_{2}(p)-v_{2}(p)\right\|^{2}\left\|D_{v_{1}}(p)+\hbar_{2}(p)-v_{2}(p)\right\|^{2}\right)}{\left\|E_{v_{2}}(p)+\hbar_{2}(p)-v_{1}(p)\right\|^{2}+\left\|D_{v_{1}}(p)+\hbar_{1}(p)-v_{2}(p)\right\|^{2}} \\
\times \sqrt{1+k_{1}^{2} e^{i \cot k_{1}}, \quad} \\
A_{4}\left(v_{1}, v_{2}\right)(p)=\max \left\{a_{1}\left(v_{1}, v_{2}\right)(p), a_{2}\left(v_{1}, v_{2}\right)(p), a_{3}\left(v_{1}, v_{2}\right)(p), a_{4}\left(v_{1}, v_{2}\right)(p)\right\},
\end{gathered}
$$


where

$$
\begin{aligned}
& a_{1}\left(v_{1}, v_{2}\right)(p)=\left\|D_{v_{1}}(p)+\hbar_{1}(p)-v_{1}(p)\right\|^{2} \sqrt{1+k_{1}^{2}} e^{i \cot k_{1}} \\
& a_{2}\left(v_{1}, v_{2}\right)(p)=\left\|E_{v_{2}}(p)+\hbar_{2}(p)-v_{2}(p)\right\|^{2} \sqrt{1+k_{1}^{2}} e^{i \cot k_{1}} \\
& a_{3}\left(v_{1}, v_{2}\right)(p)=\left\|E_{v_{2}}(p)+\hbar_{2}(p)-v_{1}(p)\right\|^{2} \sqrt{1+k_{1}^{2}} e^{i \cot k_{1}} \\
& a_{4}\left(v_{1}, v_{2}\right)(p)=\left\|D_{v_{1}}(p)+\hbar_{1}(p)-v_{2}(p)\right\|^{2} \sqrt{1+k_{1}^{2}} e^{i \cot k_{1}}
\end{aligned}
$$

Then, the two UITEs, i.e., (90), have a unique common solution.

Proof. Define $J, K, f: V \longrightarrow V$ as

$J v_{1}=J v_{1}(p)=D_{v_{1}}(p)+\hbar_{1}(p)=D_{v_{1}}+\hbar_{1}, f v_{1}=f v_{1}(p)=v_{1}(p)=v_{1}$, $K v_{2}=K v_{2}(p)=E_{v_{2}}(p)+\hbar_{2}(p)=E_{v_{2}}+\hbar_{2}, f v_{2}=f v_{2}(p)=v_{2}(p)=v_{2}$.

Then, we have the following four cases:

(1) If $A_{1}\left(v_{1}, v_{2}\right)(p)$ is the maximum term in $\left\{A_{1}\left(v_{1}, v_{2}\right)\right.$ $\left.(p), A_{2}\left(v_{1}, v_{2}\right)(p), A_{3}\left(v_{1}, v_{2}\right)(p), A_{4}\left(v_{1}, v_{2}\right)(p)\right\}$, then from (92), (93), and (99), we have that

$$
\delta\left(J v_{1}, K v_{2}\right) \leq \mu\left\|v_{1}-v_{2}\right\|^{2} \sqrt{1+k_{1}^{2}} e^{i \cot k_{1}}
$$

for all $v_{1}, v_{2} \in V$. Hence, the mappings $J, K$ and $f$ satisfy all the conditions of Theorem 9 with $\mu=\eta_{1}$ and $\eta_{2}=\eta_{3}=\eta_{4}=0$ in (6). Then, the given two UITEs, i.e., (90), have a unique common solution in $V$.

(2) If $A_{2}\left(v_{1}, v_{2}\right)(p)$ is the maximum term in $\left\{A_{1}\left(v_{1}, v_{2}\right)\right.$ $\left.(p), A_{2}\left(v_{1}, v_{2}\right)(p), A_{3}\left(v_{1}, v_{2}\right)(p), A_{4}\left(v_{1}, v_{2}\right)(p)\right\}$, then from (92), (93), and (99), we have that

$$
\delta\left(J v_{1}, K v_{2}\right) \leq \mu \frac{\left\|D_{v_{1}}+\hbar_{1}-v_{1}\right\|^{2}\left\|E_{v_{2}}+\hbar_{2}-v_{2}\right\|^{2}\left(\sqrt{1+k_{1}^{2}} e^{i \cot k_{1}}\right)^{2}}{1+\left\|v_{1}-v_{2}\right\|^{2} \sqrt{1+k_{1}^{2}} e^{i \cot k_{1}}},
$$

for all $v_{1}, v_{2} \in V$. Hence, the mappings $J, K$ and $f$ satisfy all the conditions of Theorem 9 with $\mu=$ $\eta_{2}$ and $\eta_{1}=\eta_{3}=\eta_{4}=0$ in (6). Then, the given two UITEs, i.e., (90), have a unique common solution in $V$.

(3) If $A_{3}\left(v_{1}, v_{2}\right)(p)$ is the maximum term in $\left\{A_{1}\left(v_{1}, v_{2}\right)\right.$ $\left.(p), A_{2}\left(v_{1}, v_{2}\right)(p), A_{3}\left(v_{1}, v_{2}\right)(p), A_{4}\left(v_{1}, v_{2}\right)(p)\right\}$, then from (92), (93), and (99), we have that

$$
\delta\left(J v_{1}, K v_{2}\right) \leq \mu \frac{\left(\left\|D_{v_{1}}+\hbar_{1}-v_{1}\right\|^{2}\left\|E_{v_{2}}+\hbar_{2}-v_{1}\right\|^{2}+\left\|E_{v_{2}}+\hbar_{2}-v_{2}\right\|^{2}\left\|D_{v_{1}}+\hbar_{1}-v_{2}\right\|^{2}\right) \sqrt{1+k_{1}^{2}} e^{i \cot k_{1}}}{\left\|E_{v_{2}}+\hbar_{2}-v_{1}\right\|^{2}+\left\|D_{v_{1}}+\hbar_{1}-v_{2}\right\|^{2}}
$$

for all $v_{1}, v_{2} \in V$. Hence, the mappings $J, K$ and $f$ satisfy all the conditions of Theorem 9 with $\mu=\eta_{3}$ and $\eta_{1}=\eta_{2}=\eta_{4}=0$ in (6). Then, the given two UITEs, i.e., (90), have a unique common solution in $V$.

(4) If $A_{4}\left(v_{1}, v_{2}\right)(p)$ is the maximum term in $\left\{A_{1}\left(v_{1}, v_{2}\right)\right.$ $\left.(p), A_{2}\left(v_{1}, v_{2}\right)(p), A_{3}\left(v_{1}, v_{2}\right)(p), A_{4}\left(v_{1}, v_{2}\right)(p)\right\}$, then from (93), we have that

$$
M\left(v_{1}, v_{2}\right)=A_{4}\left(v_{1}, v_{2}\right)(p),
$$

Then, there are furthermore four subcases arise:

(i) If $a_{1}\left(v_{1}, v_{2}\right)(p)$ is the maximum term in $\left\{a_{1}\left(v_{1}, v_{2}\right)\right.$ $\left.(p), a_{2}\left(v_{1}, v_{2}\right)(p), a_{3}\left(v_{1}, v_{2}\right)(p), a_{4}\left(v_{1}, v_{2}\right)(p)\right\}$. Then, from (92), (97), (99), and (103), we have that

$$
\delta\left(J v_{1}, K v_{2}\right) \leq \mu\left\|D_{v_{1}}+\hbar_{1}-v_{1}\right\|^{2} \sqrt{1+k_{1}^{2}} e^{i \cot k_{1}}
$$

for all $v_{1}, v_{2} \in V$. Hence, the mappings $J, K$ and $f$ satisfy all the conditions of Theorem 9 with $\mu=\eta_{4}$ and $\eta_{1}=\eta_{2}=\eta_{3}=$ 0 in (6). Then, the given two UITEs, i.e., (90), have a unique common solution in $\mathrm{V}$.

(ii) If $a_{2}\left(v_{1}, v_{2}\right)(p)$ is the maximum term in $\left\{a_{1}\left(v_{1}, v_{2}\right)\right.$ $\left.(p), a_{2}\left(v_{1}, v_{2}\right)(p), a_{3}\left(v_{1}, v_{2}\right)(p), a_{4}\left(v_{1}, v_{2}\right)(p)\right\}$. Then, from (92), (97), (99), and (103), we have that

$$
\delta\left(J v_{1}, K v_{2}\right) \leq \mu\left\|E_{v_{2}}+\hbar_{2}-v_{2}\right\|^{2} \sqrt{1+k_{1}^{2}} e^{i \cot k_{1}}
$$

for all $v_{1}, v_{2} \in V$. Hence, the mappings $J, K$ and $f$ satisfy all the conditions of Theorem 9 with $\mu=\eta_{4}$ and $\eta_{1}=\eta_{2}=\eta_{3}=$ 0 in (6). Then, the given two UITEs, i.e., (90), have a unique common solution in $\mathrm{V}$.

(iii) If $a_{3}\left(v_{1}, v_{2}\right)(p)$ is the maximum term in $\left\{a_{1}\left(v_{1}, v_{2}\right)\right.$ $\left.(p), a_{2}\left(v_{1}, v_{2}\right)(p), a_{3}\left(v_{1}, v_{2}\right)(p), a_{4}\left(v_{1}, v_{2}\right)(p)\right\}$.

Then, from (92), (97), (99), and (103), we have that 


$$
\delta\left(J v_{1}, K v_{2}\right) \leq \mu\left\|E_{v_{2}}+\hbar_{2}-v_{1}\right\|^{2} \sqrt{1+k_{1}^{2}} e^{i \cot k_{1}}
$$

for all $v_{1}, v_{2} \in V$. Hence, the mappings $J, K$ and $f$ satisfy all the conditions of Theorem 9 with $\mu=\eta_{4}$ and $\eta_{1}=\eta_{2}=\eta_{3}=$ 0 in (6). Then, the given two UITEs, i.e., (90), have a unique common solution in $\mathrm{V}$.

(iv) If $a_{4}\left(v_{1}, v_{2}\right)(p)$ is the maximum term in $\left\{a_{1}\left(v_{1}, v_{2}\right)\right.$ $\left.(p), a_{2}\left(v_{1}, v_{2}\right)(p), a_{3}\left(v_{1}, v_{2}\right)(p), a_{4}\left(v_{1}, v_{2}\right)(p)\right\}$. Then, from (92), (97), (99), and (103), we have that

$$
\delta\left(J v_{1}, K v_{2}\right) \leq \mu\left\|D_{v_{1}}+\hbar_{1}-v_{2}\right\|^{2} \sqrt{1+k_{1}^{2}} e^{i \cot k_{1}}
$$

for all $v_{1}, v_{2} \in V$. Hence, the mappings $J, K$ and $f$ satisfy all the conditions of Theorem 9 with $\mu=\eta_{4}$ and $\eta_{1}=\eta_{2}=\eta_{3}=$ 0 in (6). Then, the given two UITEs, i.e., (90), have a unique common solution in $\mathrm{V}$.

\section{Conclusions}

We proved some unique CFP-theorems in complex-valued b-metric spaces under the more generalized rational type contraction conditions for compatible three self-mappings in which a one self-map is continuous. Our results extend and improved many results given in the literature (e.g., see $[26,23])$. In the support of our work, we presented some illustrative examples for three self-mappings in complexvalued b-metric spaces. Moreover, we presented an application of the two UITEs to get the existing result of a common solution to support our main work. In this direction, many results can be contributed to the complex-valued b-metric spaces by using different contractive type single-valued mappings with different types of applications.

\section{Data Availability}

Data sharing is not applicable to this article as no data set were generated or analysed during the current study.

\section{Conflicts of Interest}

The authors declare that there is no conflict of interest regarding the publication of this paper.

\section{Acknowledgments}

The authors are grateful to the Deanship of Scientific Research, King Saud University for funding through Vice Deanship of Scientific Research Chairs.

\section{References}

[1] S. Banach, "Sur les opérations dans les ensembles abstraits et leur application aux équations intégrales," Fundamenta Mathematicae, vol. 3, pp. 133-181, 1922.

[2] T. M. Al-Shami, "Soft separation axioms and fixed soft points using soft semiopen sets," Journal of Applied Mathematics, vol. 2020, Article ID 1746103, 11 pages, 2020.
[3] T. M. Al-Shami and E. A. Abo-Tabl, "Soft a-separation axioms and a-fixed soft points," AIMS Mathematics, vol. 6, no. 6, pp. 5675-5694, 2021.

[4] I. A. Bakhtin, "The contraction mapping principle in almost metric spaces," Journal of Functional Analysis, vol. 30, pp. 26-37, 1989.

[5] S. Czerwik, "Nonlinear set-valued contraction mappings in bmetric spaces," Atti del Seminario Matematico e Fisico (Dell'Univ. di Modena), vol. 46, pp. 263-276, 2008.

[6] M. Akkouchi, "Common fixed point theorems for two self mappings of a b-metric space under an implicit relation," Hacettepe Journal of Mathematics and Statistics, vol. 40, no. 6, pp. 805-810, 2011.

[7] A. Aghajani, M. Abbas, and J. R. Roshan, "Common fixed point of generalized weak contractive mappings in partially ordered b-metric spaces," Mathematica Slovaca, vol. 64, no. 4, pp. 941-960, 2014.

[8] H. Aydi, M. Bota, E. Karapinar, and S. Mitrovic, "A fixed point theorem for set-valued quasi-contractions in b-metric spaces," Fixed Point Theory and its Applications, vol. 88, 2012.

[9] H. Aydi, M. Bota, E. Karapinar, and S. Moradi, "A common fixed point for weak $\phi$-contractions on b-metric spaces," Fixed Point Theory, vol. 13, no. 2, pp. 337-346, 2012.

[10] J. R. Roshan, N. Shobkolaei, S. Sedghi, and M. Abbas, "Common fixed point of four maps in b-metric spaces," Hacettepe Journal of Mathematics and Statistics, vol. 43, no. 4, pp. 613624, 2014

[11] E. Ameer, H. Aydi, M. Arshad, H. Alsamir, and M. S. Noorani, "Hybrid multivalued type contraction mapping in $\alpha_{K}$-complete partial b-metric spaces and applications," Symmetry, vol. 11, no. 1, p. 86, 2019.

[12] M. Boriceanu, "Strict fixed point theorems for multivalued operators in b-metric spaces," International Journal of Modern Mathematics, vol. 4, no. 3, pp. 285-301, 2009.

[13] M. Boriceanu, M. Bota, and A. Petrusel, "Multivalued operators in b-metric spaces," Central European Journal of Mathematics, vol. 8, no. 2, pp. 367-377, 2010.

[14] M. Bota, A. Molnar, and C. S. Varga, “On Ekeland's variational principle in b-metric spaces," Fixed Point Theory, vol. 12, pp. 21-28, 2011.

[15] S. Czerwik, "Contraction mappings in b-metric spaces," Acta Mathematica et Informatica Universitatis Ostraviensis, vol. 1, pp. 5-11, 1993.

[16] S. Czerwik, K. Dlutek, and S. L. Sing, "Round-off stability of iteration procedures for set-valued operators in b-metric spaces," Journal of Natural \& Physical Sciences, vol. 11, pp. 87-94, 2007.

[17] N. Hussain and M. H. Shah, "KKM mappings in cone b-metric spaces," Computers \& Mathematcs with Applications, vol. 62, pp. 1677-1684, 2011.

[18] E. Karapinar, S. Czerwik, and H. Aydi, “ $(\alpha, \psi)$-Meir-Keeler contraction mappings in generalized b-metric spaces," Journal of Function Spaces, vol. 2018, Article ID 326420, 4 pages, 2018.

[19] M. Samreen, T. Kamran, and N. Shahzad, "Some fixed point theorems in b-metric space endowed with graph," Abstract and Applied Analysis, vol. 2013, Article ID 967132, 9 pages, 2013.

[20] A. Azam, B. Fisher, and M. Khan, "Common fixed point theorems in complex valued metric spaces," Numerical Functional Analysis and Optimization, vol. 32, no. 3, pp. 243-253, 2011. 
[21] F. Rouzkard and M. Imdad, "Some common fixed point theorems on complex valued metric spaces," Computers \& Mathematics with Applications, vol. 64, pp. 1866-1874, 2012.

[22] S. K. Mohanta and R. Maitra, "Common fixed point of three self mappings in complex valued metric spaces," International Journal of Mathematical Archive, vol. 3, no. 8, pp. 2946-2953, 2012.

[23] W. Sintunavarat and P. Kumam, "Generalized common fixed point theorems in complex valued metric spaces and applications," Journal of Inequalities and Applications, vol. 2012, 2012.

[24] R. K. Verma and H. K. Pathak, "Common fixed point theorems using property (E.A) in complex valued metric space," Thai Journal of Mathematics, vol. 11, no. 2, pp. 347-355, 2013.

[25] K. P. R. Rao, P. R. Swamy, and J. R. Prasad, “A common fixed point theorem in complex valued b-metric spaces," Bulletin of Mathematics and Statistics Research, vol. 1, no. 1, 2013.

[26] A. A. Mukheimer, "Some common fixed point theorems in complex valued -metric spaces," The Scientific World Journal, vol. 2014, Article ID 587825, 6 pages, 2014.

[27] G. Jungck, "Compatible mappings and common fixed points," International Journal of Mathematics and Mathematical Sciences, vol. 9, Article ID 531318, 9 pages, 1986. 